KMU FORSCHUNG AUSTRIA

Austrian Institute for SME Research

\title{
Begleitende Evaluierung des „iP ImpulsProgramm creativwirtschaft"
}


Diese Studie wurde im Auftrag der ARGE iP ImpulsProgramm creativwirtschaft durchgeführt.

KMU FORSCHUNG AUSTRIA

Austrian Institute for SME Research

(Präsident: em. Univ.-Prof. Dr.Dr.h.c. J. Hanns Pichler, M.Sc.)

Für den Inhalt verantwortlich: Walter Bornett

Verfasserlinnen des Berichts:

Alfred Radauer

Aliette Dörflinger

Layout:

Susanne Fröhlich

Die vorliegende Studie wurde nach allen Maßstäben der Sorgfalt erstellt.

Die KMU FORSCHUNG AUSTRIA übernimmt jedoch keine Haftung für Schäden oder Folgeschäden die auf diese Studie oder auf mögliche fehlerhafte Angaben zurückgehen.

Dieses Werk ist urheberrechtlich geschützt. Jede Art von Nachdruck, Vervielfältigung, Verbreitung, Wiedergabe, Übersetzung oder Einspeicherung und Verwendung in Datenverarbeitungssystemen, und sei es auch nur auszugsweise, ist nur mit ausdrücklicher Zustimmung der KMU FORSCHUNG AUSTRIA gestattet. 


\section{Inhaltsverzeichnis}

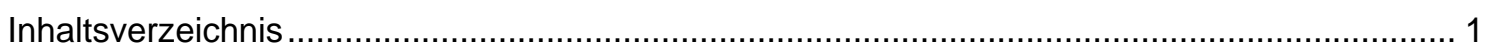

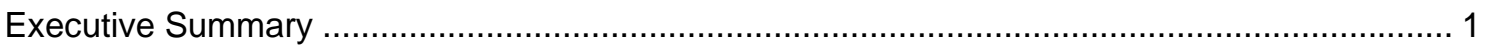

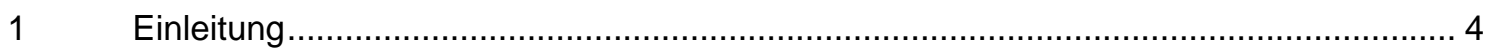

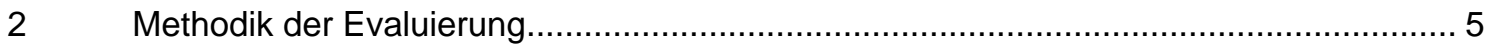

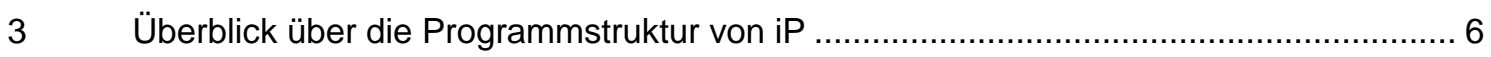

3.1 Zielsetzungen und Maßnahmen des iP ImpulsProgramm creativwirtschaft .................... 6

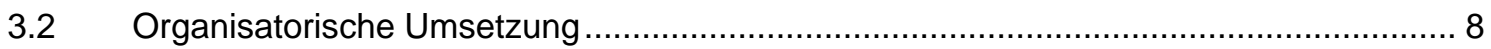

$4 \quad$ Analyse des iP ImpulsProgramm creativwirtschaft ...................................................... 10

4.1 Aspekte des generellen Programmmanagements .................................................. 10

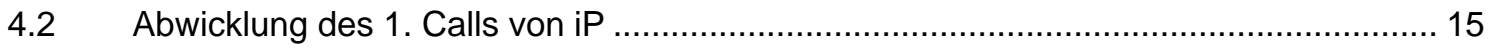

4.2.1 Der Ablauf des 1. Calls des iP ImpulsProgramm creativwirtschaft .............................. 15

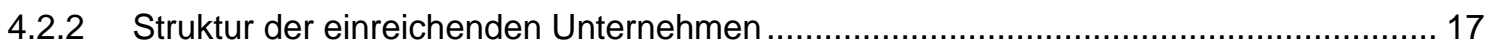

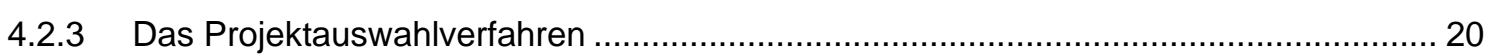

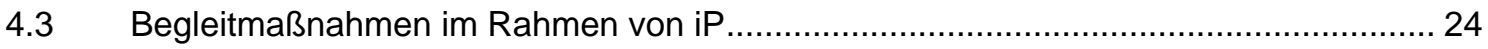

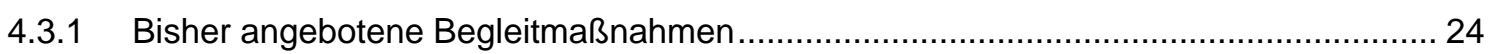

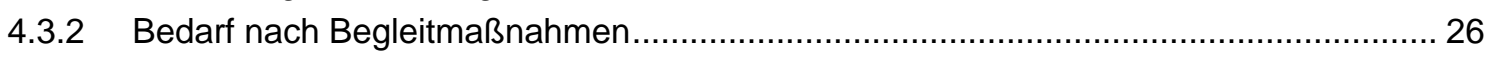

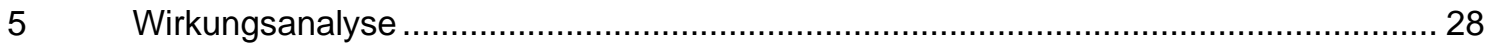

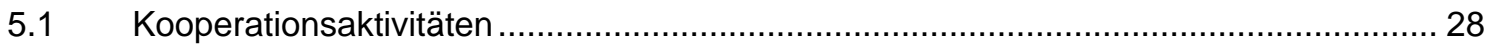

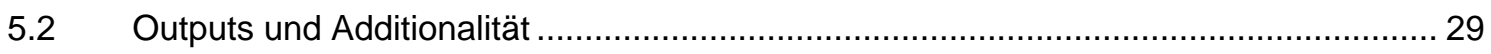

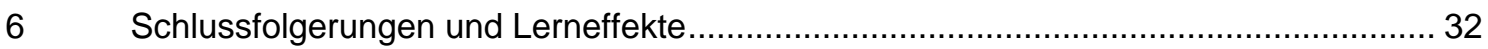

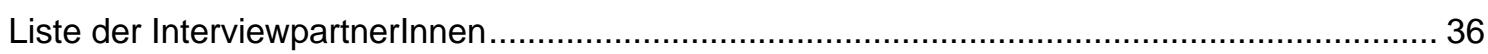




\section{Grafikverzeichnis}

Grafik 1 Informationskanäle, über die die Unternehmen vom iP ImpulsProgramm creativwirtschaft erfahren haben, Betriebe in Prozent

Grafik 2 Einschätzung der Bekanntheit von iP in der Creative Industries Community, am 1. Call teilnehmende Betriebe sowie Interessenten in Prozent

Grafik 3 Wahrnehmung der Rollen der arge creativwirtschaft und der aws hinsichtlich iP

Grafik 4 Zufriedenheit mit Aspekten des generellen Programmmanagements

Grafik 5

Zufriedenheit mit Aspekten des generellen Programmmanagements, nach Fachbereichen

Grafik 6

Großenverteilung der Unternehmen, die im Rahmen des 1. Calls von iP einen Antrag gestellt haben

Grafik $7 \quad$ Gründungsjahr der Unternehmen, die im Rahmen des 1. Calls von iP einen Antrag gestellt haben

Grafik 8 Teilnahme an anderen Förderprogrammen in den vergangenen fünf Jahren, Einreicher in Prozent

Grafik 9

Zufriedenheit mit verschiedenen Aspekten des Projektauswahlver-

fahrens, arithmetische Mittelwerte, Einreicher gesamt

Grafik $10 \quad$ Zufriedenheit mit verschiedenen Aspekten des Projektauswahlverfahrens, arithmetische Mittelwerte, nach Antragsphasen....

Grafik 11 Gründe für die Nicht-Teilnahme an den im Rahmen von iP angebotenen Businessplan-Workshops, Betriebe in Prozent.......

Grafik 12 Zufriedenheit mit verschiedenen Aspekten der im Rahmen von iP abgehaltenen Businessplan-Workshops, arithmetische Mittelwerte

Grafik 13

Nachfrage nach Aus- und Weiterbildungsmaßnahmen im Rahmen von iP, nach Themenbereichen, am 1. Call beteiligte Unternehmen in Prozent

Grafik 14

Häufigkeit, mit der bei iP einreichende Unternehmen in den vergangenen 5 Jahren Kooperationen eingegangen sind, nach Arten von Kooperationspartnern.

Grafik 15

Additionalität im 1. Call des iP ImpulsProgramm creativwirtschaft , bezogen auf 13 antwortende Unternehmen, Betriebe absolut

\section{Tabellenverzeichnis}

Tabelle 1 Rücklaufquoten der Online-Befragung der an iP teilnehmenden Unternehmen (Einreicher), nach Phase der Antragsstellung, sowie der auf der Homepage registrierten Interessenten .................................................. 5

Tabelle 2: $\quad$ Zahl der Projektanträge in den einzelnen Phasen des 1.Calls von iP ............ 16

Tabelle 3: $\quad$ Regionale Verteilung der Projektanträge nach Kurzantragsphase und

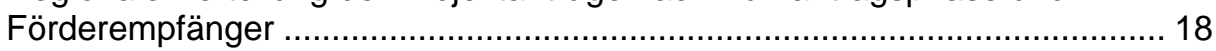

Tabelle $4 \quad$ Geplante marktorientierte Outputs in den iP Projekten ............................... 29

Tabelle 5 Geplanter Output im Bereich der Intellectual Property Rights (IPR) und im Beschäftigungsbereich 


\section{Executive Summary}

Im vorliegenden Bericht werden die Ergebnisse der begleitenden Evaluierung des Programms iP ImpulsProgramm creativwirtschaft (iP), die von der KMU FORSCHUNG AUSTRIA im Auftrag der ARGE iP ImpulsProgramm creativwirtschaft durchgeführt wurde, dargestellt. Ziel der Evaluierung war, das Programm hinsichtlich seines bisherigen Verlaufs zu bewerten, Verbesserungspotenzial zu identifizieren und entsprechende Maßnahmenvorschläge zu erarbeiten. Für die Evaluierung wurde methodisch auf eine Dokumentenanalyse, eine Online Befragung bei allen etwa 470 an iP teilnehmenden Betrieben und auf der Homepage registrierten Interessenten sowie 17 qualitative Interviews mit Programmverantwortlichen, Stakeholdern, iP MitarbeiterInnen, Jurorlnnen und externen Experten, zurückgegriffen.

Die Evaluierungsergebnisse führen insgesamt zu dem Befund, dass das „iP ImpulsProgramm creativwirtschaft" die zugedachte Rolle als Impulsbringer für die österreichische Creative Industries Community überwiegend erfüllt und in einigen Bereichen die Erwartungen auch übertrifft. Positiv hervorzuheben sind $\mathrm{u}$. a. die hohe Anzahl an Einreichungen im 1. Call, die Breite angesprochener Unternehmen (neu gegründete wie auch bestehende Betriebe, siehe hierzu weiter unten) oder die hohen Zufriedenheitswerte mit dem Programmmanagement. Zudem bestehen Hinweise auf vergleichsweise geringe Mitnahmeeffekte bei den geförderten Projekten. In bestimmten (Detail-)bereichen zeigt sich allerdings auch Optimierungspotenzial. Dies betrifft in erster Linie eine klarere Ziel-Maßnahmendefinition (hier ist vor allem das immanente Spannungsfeld zwischen „Kreativitäts“- und „technologisch orientierter Innovationsförderung" zu erwähnen; auch hierzu unten mehr) und die Transparenz bei der Bewertung der Projektanträge.

Im Einzelnen ergibt sich folgendes Bild:

Das iP ImpulsProgrammm creativwirtschaft ist ein bundesweit operierendes Förderprogramm, das die Stärkung des Innovationspotenzials von KMU in der Kreativwirtschaft als Globalziel definiert. Des Weiteren soll das Programm dazu beitragen, Österreich als international wettbewerbfähigen Kreativstandort zu positionieren. Um diese Globalziele wie auch weitere Zwischenziele zu erreichen, greift das Programm auf Instrumentenebene hauptsächlich auf eine finanzielle Förderung, die so genannte iP Förderung, zurück. Diese wird im Rahmen von Calls ausgeschüttet. Gefördert werden innovative Einzelprojekte mit Bezug zur Kreativwirtschaft. Der Zuschuss beträgt maximal $70 \%$ der Projektkosten bei einem Maximalbetrag (auf Grund der „de minimis" Regelung) von € 100.000,-. Ergänzend werden Begleitmaßnahmen im Bereich Ausund Weiterbildung angeboten. Gegenüber ähnlichen Programmen grenzt sich das Programm durch seine Fokussierung auf technisch-orientierte Innovationen bei gleichzeitiger Berücksichtigung des Kreativitätsgehaltes bzw. des Bezugs zur Kreativwirtschaft ab. Thematisch beschränkt sich iP auf die drei Bereiche Design, Multimedia und Musik. Träger des Programms sind die Austria Wirtschafts Service Gesmbh (aws) und die arge creativwirtschaft der Wirtschaftskammer Österreich, die beide für die Durchführung des Programms eine Arbeitsgemeinschaft, die ARGE iP ImpulsProgramm creativwirtschaft, gegründet haben. Die operativen Tätigkeiten obliegen großteils dem iP Büro der ARGE, das seinen Sitz im Wiener Museumsquartier hat und als Anlaufstelle für Interessenten und Antragssteller fungiert.

Bislang wurde ein Call im Zeitraum von Dezember 2004 bis Juni 2005 abgewickelt. Die Anzahl der Anträge (315) übertraf die Erwartungen des Programmmanagements, wobei in einem zweistufigen Verfahren 24 Projekte schließlich zur Förderung ausgewählt wurden. Der zweite Call läuft seit Mitte Dezember 2005. Für beide Calls wurden insgesamt $€$ 4,8 Mio (inklusive der Abwicklungsgemeinkosten) budgetiert. Die Betriebe, die im Rahmen des 1. Calls einen Antrag gestellt haben, sind überwiegend klein(st)betrieblich strukturiert und jung: Nur $48 \%$ der Unternehmen sind älter als zwei Jahre. Etwa $28 \%$ der Unternehmen wurde anlässlich der Teilnahme an iP gegründet, wobei die meisten dieser Gründungen auf den Bereich Design entfielen, gefolgt vom Bereich Musik und dem Bereich Multimedia. Insgesamt zeigt sich, dass iP 
eine hohe Breite an bestehenden und neu gegründeten Unternehmen erreicht hat, womit das Programm einerseits seine Impulsfunktion im Gründungsbereich erfüllt, ohne gleichzeitig bestehende Unternehmensgründungsprogramme zu duplizieren, andererseits aber auch die Erfahrung (und auch Ansatzpunkte für die Förderung) bestehender Betriebe berücksichtigt. Dies ist positiv zu werten und grenzt iP gegenüber eher gründungsorientierten Programmen im deutschsprachigen Ausland ab.

Es zeigt sich weiters, dass bis zu $40 \%$ der Einreicher Erfahrungen mit Förderprogrammen haben - hauptsächlich im Bereich der Innovationsförderung (Stichwort: Projektförderungen des ehemaligen Forschungsförderungsfonds (FFF) und dem nunmehrigen Bereich Basisprogramme der Forschungsförderungsgesellschaft (FFG), etwas weniger (und vor allem auf Wien beschränkt) im Bereich der Kreativwirtschaftsförderung (Stichwort: departure) und kaum im Bereich der reinen Kunst- und Kulturförderung. Dies deutet darauf hin, dass iP die Zielgruppe der Kreativen nur teilweise angesprochen hat und dass bei iP vermutlich vergleichsweise viele Unternehmen, die kaum einen Bezug zur Kreativwirtschaft haben und iP nur als alternatives Innovationsförderprogramm für technische Produktentwicklungen ansehen, Förderanträge stellen.

Untermauert wird dieser Eindruck auch durch die Inhalte der Projektanträge, die in einigen Fällen nur wenig kreativ-künstlerische Elemente erkennen ließen. Generell bewegt sich iP durch seine Abgrenzung in einem Spannungsfeld zwischen „Kreativitätsförderung" und „technisch orientierter Innovationsförderung", wobei die Festlegung (und vor allem die anschließende Kommunikation dieser Festlegung an die Unternehmen) des Stellenwertes des Kreativitätsaspektes verbesserungswürdig erscheint.

Ein wesentliches Problemfeld, das sich in diesem Zusammenhang sowie hinsichtlich des Projektauswahlverfahrens ergibt, ist das der Transparenz der Projektbeurteilungskriterien. Von allen Aspekten im Zusammenhang mit dem Projektauswahlverfahren weist die Transparenz am meisten Verbesserungspotenzial auf. Dies lässt sich auf drei Faktoren zurückführen: Erstens, die bereits beschriebenen Unschärfen in der Kommunikation der Programmabgrenzungskriterien. Dies betrifft nicht nur die Diskussion über den Kreativitätsgehalt, sondern auch das Ziel-Maßnahmensystem von iP, wo vereinzelt Subziele (speziell das Kooperationsziel) keine oder nicht hinreichend Entsprechung auf Instrumentenebene haben. Zweitens, die Unzufriedenheit der Betriebe mit den ihrer Meinung nach zu standardisierten Absagebriefen. Und drittens das thematische Setting des Programms, da eine "objektive“ Beurteilung kreativkünstlerischer Elemente immer mit besonderen Schwierigkeiten verbunden ist.

Neben der Transparenz zeigt sich ein negativer Bias auch gegenüber dem Aufwand für die Bereitstellung betriebswirtschaftlicher Daten im Projektantrag. Hier dürften vor allem neu gegründete Betriebe überfordert gewesen sein. Ansonsten werden die anderen Aspekte des Projektauswahlverfahrens relativ positiv beurteilt, wie z. B. die weitere Abwicklung der geförderten Projekte durch die aws oder allgemein die Zeit, die für die Erstellung der Anträge zur Verfügung stand. Noch besser wird das allgemeine Programmmanagement bewertet. Dem Arbeitseinsatz des iP Büros, der Öffentlichkeitsarbeit oder der Gestaltung der Informationsunterlagen wird allesamt ein gutes Zeugnis ausgestellt. Im Bereich der PR Aktivitäten wurde vereinzelt angemerkt, dass mehr konkrete Programminhalte kommuniziert werden sollten. Insgesamt ist es im Rahmen der PR Tätigkeiten innerhalb kurzer Zeit gelungen, eine vergleichsweise hohe Zahl an Betrieben anzusprechen. Auch der erreichte Bekanntheitsgrad dürfte relativ hoch, wenngleich sicher ausbaufähig sein. Primär genutzter Verbreitungskanal ist das Internet, allerdings kommt informellen Kontakten und den Roadshows (diesen aber hauptsächlich auf Grund von Multiplikatoreffekten) ebenfalls eine bedeutende Rolle zu.

Im Rahmen der Begleitmaßnahmen wurde bislang ein relativ gut besuchter Businessplanworkshop angeboten, mit dem die Unternehmen auch durchaus zufrieden waren. Einzig eine stärkere Berücksichtigung von Spezifika der Kreativwirtschaft (und damit verbunden ein höherer Neuigkeitswert der Seminarinhalte) wurde vereinzelt angeregt. Die Begleitmaßnahmen weisen darüber hinausgehend innerhalb von iP Ausbaupotenzial auf. Zum Einen dürften viele Unternehmen, speziell im Bereich Design und noch mehr im Bereich Musik, mit der Erstellung 
fundierter Businesspläne und Marktanalysen Schwierigkeiten gehabt haben - was sich auch in der Qualität der Anträge niedergeschlagen hat. Dies ist eine Folge eines im Vergleich zu traditionellen Wirtschaftssektoren unterschiedlichen Unternehmensverständnisses und somit letztlich strukturell vorgegeben. Zum anderen artikulieren die Betriebe selbst erheblichen Bedarf an Aus- und Weiterbildungsmaßnahmen. Hier gilt es, angesichts knapper Ressourcen, eine Priorisierung vorzunehmen und Synergiepotenziale mit etablierten Anbietern von Aus- und Weiterbildungsmaßnahmen, so fern möglich, zu nutzen.

Auf Grund der kurzen Laufzeit waren im Rahmen dieser Evaluierung noch keine konkreten Outputs aus den Projekten zu verzeichnen. Die diesbezüglichen Erwartungen der Betriebe dürften - speziell hinsichtlich der Zahl der anzumeldenden Patente - jedoch sehr ambitioniert sein. Weiters gibt es Hinweise darauf, dass diese Outputs ohne Förderung durch iP nicht oder nur im reduzierten Umfang zustande kommen würden. Die diesbezüglich ermittelten Additionalitätswerte sind jedoch nur indikativ zu sehen, da eine adäquate Kontrollgruppe nicht zur Verfügung gestanden ist und die Zahl der auf diese Frage antwortenden Unternehmen im Verhältnis zur ebenfalls niedrigen Gesamtzahl der Fördernehmer gering war. Einen Zusatznutzen von iP sehen die Betriebe vor allem in einer gesteigerten Publicity und einem besseren Unternehmensimage. Auch hier spiegelt sich eine hohe Erwartungshaltung bei der Zielgruppe hinsichtlich zukünftiger iP Aktivitäten sowie des Renommees des Programms wider. $64 \%$ der Einreicher würden wieder einen Antrag im Rahmen von iP stellen, während nur $6 \%$ davon dezidiert absehen.

Trotz der aufgezeigten positiven Effekte erscheint es (zumindest aus mittel- und langfristigen Überlegungen heraus) wichtig zu unterstreichen, dass die identifizierten Verbesserungspotenziale, und hier insbesondere die Transparenz, ernst genommen werden. Eine Liste an entsprechenden Maßnahmenvorschlägen findet sich in Kapitel 6 dieses Berichtes. 


\section{Einleitung}

Wenngleich sich die Definition des Begriffs der Kreativwirtschaft (bzw. der Creative Industries) je nach verwendeter Literaturquelle in Details unterscheidet, so kann die Kreativwirtschaft im Kernbereich doch als Querschnittsbranche angesehen werden, bei der künstlerisch-kreative Elemente eine wesentliche Rolle für die Leistungserstellung einnehmen. Wesentliches Kennzeichen der Kreativwirtschaft ist das identifizierte Wachstums- und Beschäftigungspotenzial. So ist in Österreich die Zahl der Betriebe in den Creative Industries im Zeitraum 1995 bis 2000 um etwa $33 \%$ auf ca. 20.900 Unternehmen gestiegen. Die Zahl der Beschäftigten stieg im selben Zeitraum auf rd. 129.500, was einem Plus von $29 \%$ entspricht $^{1}$. Das Bild von innovativen, dynamischen Unternehmen prägt diesen Bereich auch im internationalen Kontext.

Vor diesem Hintergrund wurde im Jahr 2004 in Österreich das „iP ImpulsProgramm creativwirtschaft" ins Leben gerufen. Finanziert wird das Programm durch die Nationalstiftung für Forschung, Technologie \& Entwicklung, als Träger des Programms fungieren die Austria Wirtschafts Service GesmbH (aws) und die arge creativwirtschaft der Wirtschaftskammer Österreich. Ziel des Programms ist die Wettbewerbsfähigkeit und das Innovationspotenzial von $\mathrm{KMU}$, die in der Kreativwirtschaft tätig sind, zu stärken. Die Erhöhung der österreichischen Wertschöpfung in den Kreativbranchen sowie die Vernetzung und die Kooperationsaktivitäten der geförderten Betriebe können als zusätzliche Ziele genannt werden. Österreichische Unternehmen aus den Bereichen Design, Multimedia und Musik konnten bzw. können im Rahmen von Calls Anträge für die finanzielle Förderung von Einzelprojekten stellen. Weiters kommen Qualifizierungsmaßnahmen als Förderinstrumentarium zur Anwendung. Im Rahmen des ersten Calls haben 315 Unternehmen einen Kurzantrag für eine Förderung gestellt. Ende Juni 2005 wurde insgesamt 24 Projekte eine Förderzusage erteilt, wobei sich die ausgeschütteten Fördermittel auf insgesamt € 2,1 Mio belaufen. Für den 2. Call, der im Zeitraum Mitte Dezember 2005 bis Mitte Jänner 2006 durchgeführt wird, stehen weitere $€$ 2,7 Mio an Fördermitteln zur Verfügung.

Bei dem vorliegenden Bericht handelt es sich um eine begleitende Evaluierung des iP ImpulsProgramm creativwirtschaft austria, die von der KMU FORSCHUNG AUSTRIA im Auftrag der austria wirtschaftsservice $\mathrm{GmbH}$ und der arge creativwirtschaft Wirtschaftkammer Österreich im Zeitraum August 2005 bis Jänner 2006 durchgeführt wurde. Der Schwerpunkt der Evaluierung wurde auf die Bewertung des Programmablaufs und hier speziell auf die Bewertung der Abläufe im 1. Call gelegt. Aus den diesbezüglichen Erfahrungen wurden Adaptierungs- und Optimierungsvorschläge für die Programmkonzeption und weitere operative Programmtätigkeiten abgeleitet. Die Evaluierung beruht sowohl auf quantitativen als auch qualitativen Analysen unterschiedlicher Aspekte des iP Förderprogramms.

Der Bericht gliedert sich in insgesamt fünf Abschnitte bzw. Kapitel:

- Kapitel 2 stellt die methodische Vorgehensweise dar.

- Kapitel 3 bietet einen Überblick über die Programmstruktur von iP.

- Kapitel 4 befasst sich mit der Analyse des iP ImpulsProgramm creativwirtschaft, speziell hinsichtlich genereller Aspekte des Programmmanagements, der Abwicklung des 1. Calls und der Begleitmaßnahmen im Rahmen von iP.

- Kapitel 5 dient der Erörterung der bisher feststellbaren und erwarteten Wirkungen des Impulsprogramms.

- In Kapitel 6 werden schließlich die Ergebnisse der Evaluierung kurz zusammengefasst und Schlussfolgerungen und Lerneffekte abgeleitet.

\footnotetext{
${ }^{1}$ Vgl. Erster Österreichischer Kreativwirtschaftsbericht, 2003.
} 


\section{Methodik der Evaluierung}

Für die begleitende Evaluierung des „iP ImpulsProgramm creativwirtschaft“ (iP) kam ein Mix aus qualitativen und quantitativen Methoden zur Anwendung. Ausgangspunkt der Untersuchungen bildete eine Dokumentenanalyse, die einer ersten Erfassung, Abgrenzung und Positionierung der Programmaktivitäten diente. Im Rahmen von insgesamt 13 persönlichen und offenen Interviews wurden in der Folge der bisherige Verlauf der Initiative und mögliche Stärken und Schwächen erörtert sowie entsprechende Optimierungsvorschläge diskutiert. Befragt wurden hierzu VertreterInnen der Träger der Initiative, des Programmmanagements, sonstiger Stakeholder sowie auch eine Auswahl an Jurorlnnen aus allen drei Themengebieten des 1 . Calls von iP. Zur Berücksichtigung einer externen bzw. auch internationalen Sichtweise wurden darüber hinaus auch vier Experten aus dem Bereich der Kreativwirtschaft und Kreativwirtschaftsförderung befragt, die nicht an der Konzeption, Umsetzung oder Abwicklung von iP mitgewirkt haben. Eine Liste der InterviewpartnerInnen findet sich im Anhang zu dem Bericht.

Das Kernelement der Evaluierung bildete die im September/Oktober 2005 online durchgeführte Unternehmensbefragung (bzw. genauer Zielgruppenbefragung). Diese richtete sich (als Vollerhebung konzipiert) an alle Betriebe, die im Rahmen des 1. Calls von iP einen Förderantrag gestellt haben sowie an alle Personen, die sich auf der iP Homepage registrieren ließen. Unternehmen, die bis zum 10. Oktober 2005 nicht geantwortet hatten, wurden ein zweites Mal angeschrieben und teilweise zusätzlich telefonisch kontaktiert, um den Rücklauf zu maximieren. Tabelle 1 zeigt den letztlich erzielten Rücklauf, differenziert nach Phasen der Antragsstellung (Unternehmen, die lediglich einen Kurzantrag gestellt haben; Unternehmen, die einen Langantrag gestellt haben, aber keine Förderzusage erhalten haben; Förderempfänger) sowie für die auf der Homepage registrierten Interessenten.

Tabelle 1 Rücklaufquoten der Online-Befragung der an iP teilnehmenden Unternehmen (Einreicher), nach Phase der Antragsstellung, sowie der auf der Homepage registrierten Interessenten

\begin{tabular}{l|c|c|c}
\hline & $\begin{array}{c}\text { Ausgesendete } \\
\text { Fragebögen }\end{array}$ & $\begin{array}{c}\text { Auswertbare } \\
\text { Fragebögen }\end{array}$ & Rücklaufquote [\%] \\
\hline Interessenten & 184 & 25 & 13,6 \\
Kurzantragsphase & 234 & 50 & 21,4 \\
Langantragsphase & 28 & 17 & 60,7 \\
Förderempfänger & 24 & 16 & 66,7 \\
\hline \hline Gesamt & 470 & 108 & 23,0 \\
\hline Quelle: KMU FORSCHUNG AUSTRIA & & & \\
\hline
\end{tabular}

Aus den gesammelten Daten wurden schließlich - im Sinne einer formativen Evaluierung Lerneffekte erarbeitet und Maßnahmenempfehlungen abgeleitet. 


\section{3 Überblick über die Programmstruktur von iP}

\subsection{Zielsetzungen und Maßnahmen des iP ImpulsProgramm creativwirtschaft}

Ausgehend von einer Initiative der arge creativwirtschaft der Wirtschaftskammer Österreich, die sich hierzu auf das im Ersten Österreichischen Kreativwirtschaftsbericht festgestellte Wachstumspotenzial in den Creative Industries berief, wurde das „IP ImpulsProgramm creativwirtschaft" am 24. Juni 2004 per Beschluss der Nationalstiftung für Forschung, Technologie und Entwicklung ins Leben gerufen. Für die Durchführung von zunächst zwei Calls wurden Stiftungsmittel im Ausmaß von insgesamt $€ 4,8$ Mio ( $€ 2,1$ Mio für den ersten Call, $€$ 2,7 Mio für den zweiten Call) bereitgestellt. Die Finanzierung (bzw. die Programmfortführung) unterliegt einer jährlichen Beschlussfassung, eine exakte Laufzeit ist somit nicht vorgegeben.

Als Globalziel bzw. Mission des Programms kann die „Stärkung des Innovationspotenzials von bestehenden und neu gegründeten kleinen und mittleren Unternehmen...in der Kreativwirtschaft" angesehen werden, um "den Kreativanteil in der österreichischen Wirtschaft zu heben“, die "Wertschöpfung von Produkten und Dienstleistungen nachhaltig zu erhöhen" und "Österreich als einen international wettbewerbsfähigen Kreativstandort zu positionieren“. ${ }^{2}$

Dieser Mission sind eine Reihe von Sub- bzw. Zwischenzielen vorgelagert, die zwar taxativ angeführt, allerdings - auf Grund bislang fehlender Erfahrungen - nicht quantifiziert wurden. Die Zwischenziele umfassen:

- eine Erhöhung der Anzahl an Kreativwirtschaftsunternehmen in Österreich, insbesondere durch Neugründungen;

- $\quad$ eine Erhöhung der Zahl der Arbeitsplätze in den Kreativbranchen;

- die Anregung von Kooperationen zwischen Kreativwirtschaftsunternehmen einerseits untereinander und andererseits mit der „traditionellen“ Wirtschaft;

- die Stimulation von materiellen und immateriellen Investitionen;

- die Etablierung Österreichs als Kreativstandort im internationalen Umfeld, wobei hierzu mit den Außenwirtschaftsstellen der WKO kooperiert werden soll;

- eine optimale Betreuung potenzieller Gründer und Unternehmen im Kreativwirtschaftsbereich (durch diverse Beratungsleistungen, Ausbildungsmaßnahmen und Förderungen für Kreative in Kooperation mit dem Netzwerk der Wirtschaftskammern, deren Beratungs- und Ausbildungseinrichtungen, der aws etc.);

Zur Erreichung der genannten Zielsetzungen greift das iP ImpulsProgramm creativwirtschaft auf Maßnahmen- bzw. Instrumentenebene primär auf einen finanziellen Zuschuss für Einzelprojekte, der so genannten iP Förderung, zurück, die im Rahmen von Calls ausgeschüttet wird. Der Zuschuss wird für innovative Projekte mit Bezug zur Kreativwirtschaft gewährt. Flankierend werden auch Begleitmaßnahmen, speziell im Bereich Aus- und Weiterbildung, angeboten. Im Rahmen des 1. Calls wurde hierzu ein Workshop zur Erstellung von Businessplänen durchgeführt.

Auf Grund der thematischen Breite der Kreativwirtschaft beschränkt sich die iP Förderung (sowohl im ersten, als auch im zweiten Call) auf Unternehmen bzw. Projekte in den drei Bereichen Multimedia, Design und Musik. Damit folgt iP der Empfehlung von Experten, die diesen drei Feldern in der Programmkonzeptionierungsphase das höchste Entwicklungspotenzial im österreichischen Kontext zugesprochen haben. Allerdings verweisen einige der Experten auch

Vgl. ARGE iP ImpulsProgramm creativwirtschaft, PDF Gesamtdokument der Homepage, 2005, S. 5f., http://www.impulsprogramm.at, abgefragt am 10.8.2005. 
darauf, dass - vor allem im internationalen Kontext - Schnittstellenprojekte zwischen den drei Bereichen an Bedeutung gewinnen. Daher erscheint es sinnvoll, das thematische Setting von iP regelmäßig (z. B. in den Beiräten) hinsichtlich seiner Passgenauigkeit auf die österreichische Kreativwirtschaftsszene zu überprüfen.

Gegenüber anderen Förderprogrammen grenzt sich iP durch eine Reihe verschiedener Punkte ab. So versteht sich iP nicht als Programm zur Kunst- und Kulturförderung: Im Vordergrund steht vielmehr die wirtschaftliche Verwertbarkeit technologisch-innovationsorientierter Projekte, die zudem nur von Unternehmen und nicht von Einzelpersonen eingereicht werden können. Die Abgrenzung gegenüber Innovationsförderprogrammen ergibt sich wiederum durch die höhere Beachtung des Kreativitätsaspektes und gleichzeitig geringeren Anforderungen hinsichtlich des Projektinnovationsgehaltes.

Im Vergleich zu ähnlich gelagerten Förderprogrammen mit Fokus auf den Creative Industries (in diesem Zusammenhang ist vor allem das Wiener Förderprogramm departure zu nennen) ergibt sich ein Alleinstellungsmerkmal durch den bundesweiten Geltungsbereich von iP. Zudem bestehen thematische Unterschiede (departure inkludiert neben Multimedia, Design und Musik auch den Bereich Mode) und Unterschiede beim genutzten Förderinstrumentarium (Calls vs. der Möglichkeit, jederzeit während der Laufzeit Anträge stellen zu können).

Das gewählte Ziel- und Maßnahmensystem dürfte in seiner Gesamtkonzeption im Hinblick auf das Globalziel kohärent aufgebaut sein, weist aber in Deteilbereichen Unschärfen auf:

- Einigen Zwischenzielen (z. B. der Erhöhung der Kooperationsaktivitäten innerhalb der Creative Industries und mit traditionellen Branchen) scheinen bislang nicht im hinreichenden Ausmaß Instrumente zugeordnet zu sein. Vor dem Hintergrund der vielfältigen Möglichkeiten, explizit Kooperationen zu fördern (z.B. Verbundprojekte, Cluster etc.) könnten hier Erwartungen geweckt werden, die von iP in der derzeitigen Form nur schwer erfüllbar sind. Tatsächlich ist eine auf iP zurückzuführende Intensivierung der Kooperationsaktivitäten bei den geförderten Betrieben kaum zu beobachten (siehe Abschnitt 5.1).

- Bislang gab es - im Rahmen von iP - noch keine nennenswerten Aktivitäten im Zusammenhang mit den Außenwirtschaftsstellen (AWOs) der WKO.

- Die Abgrenzung zu Innovationsförderprogrammen (Betonung des Kreativitätsaspektes sowie der Kreativwirtschaft als Zielgruppe) und zu Kultur- und Kunstförderprogrammen (Technologie- und Innovationsorientierung, wirtschaftliche Verwertung) erzeugt ein Spannungsfeld, in dem sich die Frage nach dem zu berücksichtigenden Stellenwert des Kreativanteils der in den Calls eingereichten Projekte stellt. Dabei bewegt sich iP zwischen zwei Polen: Auf der einen Seite sind zwar rein künstlerische oder kulturelle Projekte von der Förderung ausgeschlossen. Auf der anderen Seite besteht jedoch die Gefahr, dass iP bei Förderung von Projekten mit zu geringem Kunst-/Kulturbezug lediglich bestehende Innovationsförderprogramme dupliziert. Letzteres könnte dazu führen, dass vermehrt Unternehmen aus traditionellen Branchen (hier spielt auch die uneinheitliche Definition der Creative Industries als Querschnittsbranche mit hinein) iP als zusätzliche alternative Förderschiene für reine Produktentwicklungen ansehen und anderweitig abgelehnte Anträge nur leicht modifiziert bei iP wieder einreichen. Weiters wäre denkbar, dass Kreative bei Durchsicht der bewilligten Projekte kaum Berührungspunkte zwischen ihren Ideen und Tätigkeiten sowie dem Förderprogramm herstellen können und infolgedessen von der Erstellung eines Antrags, z. B. im Rahmen eines späteren Calls, absehen. 


\subsection{Organisatorische Umsetzung}

Träger des iP ImpulsProgramms creativwirtschaft sind die Austria Wirtschaftsservice GmbH (aws) und die arge creativ wirtschaft austria der Wirtschaftskammer Österreich. Die aws übernimmt - auf Grund Ihrer Expertise mit der Abwicklung von Förderprogrammen als Spezialbank der Republik Österreich - für iP die Rolle der Förderabwicklungsstelle. Im Speziellen ist sie für die betriebswirtschaftliche Beurteilung der eingereichten Projekte und - nach Erteilung der Förderzusage - für die formelle Abwicklung der Förderung (finanzielles Reporting, Auszahlung etc.) zuständig. Die 2003 gegründete arge creativwirtschaft stellt fachlichen Input als Plattform und Lobbying-Organisation durch die Nutzung der geschaffenen Netzwerke in der Kreativwirtschaft zur Verfügung. Als weiterer wichtiger Stakeholder fungiert die Nationalstiftung für Forschung, Technologie und Entwicklung, die die Budgetmittel zur Verfügung gestellt hat, sich ansonsten aber nicht an der Umsetzung und Abwicklung des Programms beteiligt.

Die aws und die arge creativwirtschaft haben für Durchführung des Impulsprogramms eine eigene Arbeitsgemeinschaft, die ARGE iP ImpulsProgramm creativwirtschaft (ARGE iP), gegründet. ${ }^{3}$ Die ARGE iP stellt den organisatorischen Rahmen für iP dar.

Die ARGE iP verfügt über zwei Organe, den ARGE-Rat und die Geschäftsführung. Als oberstes Organ ist der ARGE-Rat für sämtliche Entscheidungen, die grundsätzlich-strategische Fragestellungen zu iP betreffen, zuständig. Darüber hinaus kann der ARGE Rat festlegen, dass bestimmte Themenbereiche seiner Beschlussfassung unterliegen. Der ARGE-Rat besteht aus vier Personen, wobei jeweils zwei die Wirtschaftskammer Österreich und die aws vertreten.

Dem ARGE-Rat als Aufsichtsorgan ist auf operativer Ebene die Geschäftsführung von iP unterstellt. Die Geschäftsführung setzt sich aus zwei Mitgliedern zusammen, wobei eine Person von der aws und eine von der Wirtschaftskammer Österreich nominiert wird. Beide GeschäftsführerInnen vertreten die ARGE nach außen hin gemeinsam, wobei allerdings bei Bedarf auch eine Einzelvertretungsbefugnis eingeräumt werden kann. Eine Aufgabenteilung besteht insofern, als dass die Geschäftsführerin der aws für die strategischen Belange zur Weiterentwicklung des Programms zuständig ist, während sich der von der Wirtschaftskammer bereitgestellte Geschäftsführer für die kaufmännischen und operationellen Belange des Impulsprogramms verantwortlich zeichnet. Bestimmte Aktivitäten, wie Budgetänderungen und -planungen oder die Einstellung von MitarbeiterInnen, sind an die Zustimmung des ARGE-Rates gebunden.

Für die operative Umsetzung der Initiative wurde eine eigene Anlaufstelle, das so genannte iPBüro, im November 2004 im Museumsquartier in Wien eingerichtet. Das Büro dient der Beratung und Information potenzieller FörderansucherInnen und ist für die administrative Abwicklung der Calls sowie die Organisation und Administration der PR-, Ausbildungs- und sonstiger etwaiger Begleitmaßnahmen zuständig. Zudem werden die Jurymitglieder auf Vorschlag des iP Büros ausgewählt. Das iP Büro beschäftigt drei Personen: Eine Projektleiterin sowie zwei weitere Mitarbeiterinnen, die jeweils für die Buchhaltung und die online Einreichung sowie für den Webauftritt und Aus- und Weiterbildungsfragen für die Unternehmen zuständig sind.

Neben diesen primären Organisationseinheiten wurden drei Expertenbeiräte für die Themenbereiche Design, Multimedia und Musik eingerichtet. Die Expertenbeiräte hatten ihre konstituierenden Sitzungen zwischen dem 12. September 2005 und 19. Oktober 2005. Der Aufgabenbereich der Expertenbeiräte umfasst die begleitende inhaltliche Beratung des iP Teams hinsichtlich der Gestaltung des zweiten Calls, Beratung hinsichtlich einer möglichst zielgruppenspezifischen Unternehmensansprache sowie die laufende Diskussion möglicher Optimierungen

Vgl. Arbeitsgemeinschaftsvertrag zwischen der arge creativwirtschaft der Wirtschaftskammer Österreich und der Austria Wirtschaftsservice GesmBh, 2004. 
hinsichtlich des Projektselektionssprozesses. Den Beiräten gehören etwa vier bis fünf Experten an, wobei sich ein Teil der Beiräte aus ehemaligen Juroren des 1. Calls zusammensetzt.

Bezüglich der Kommunikationsstrukturen ist festzustellen, dass auf Grund der relativ geringen Größe des iP Teams die interne Kommunikation vor allem auf informellem Weg erfolgt. Auch die Aufgabenteilung zwischen den handelnden Akteuren wird bei Bedarf flexibel gehandhabt. Nichtsdestotrotz findet etwa alle zehn Tage ein Jour-fixe statt, bei dem sich die Projektleiterin des iP Büros und die beiden GeschäftsführerInnen bezüglich der zu setzenden Aktivitäten absprechen. Sitzungen des ARGE-Rates finden entweder auf Antrag der Geschäftsführung oder bei „sonstigem“ Bedarf statt. 


\section{Analyse des iP ImpulsProgramm creativwirtschaft}

\subsection{Aspekte des generellen Programmmanagements}

Die im ARGE Vertrag festgelegten Tätigkeitsfelder erlauben eine prinzipielle Unterscheidung zwischen callspezifischen Tätigkeiten und Aktivitäten des generellen Programmmanagements. Letztere umfassen hierbei in erster Linie die awarenessbildenden Maßnahmen bzw. die Öffentlichkeitsarbeit für das Programm, aber auch die Koordination der Aktivitäten mit Programmen der Stadt Wien und der Bundesländer sowie allgemeine (im Vertrag nicht explizit erwähnte) administrative Tätigkeiten.

Im Kontext des generellen Programmmanagements nimmt die Unternehmensansprache vor allem aus zwei Gründen eine bedeutende Rolle ein. Einerseits stand wegen des vorgegeben Zeitrahmens zur Durchführung des 1. Calls (unter Berücksichtigung der damit verbundenen Themendefinitionen und des Aufbaus der für die Programmabwicklung notwendigen Infrastruktur) relativ wenig Zeit für die Entwicklung einer PR Strategie zur Verfügung. Andererseits konnte das iP ImpulsProgramm creativwirtschaft als erstes bundesweit agierendes Förderprogramm in diesem Themenfeld nur auf vergleichsweise geringe Erfahrungen mit der avisierten Zielgruppe zurückgreifen. So dürften sich z. B. viele Kreative, die Produkte und Dienstleistungen anbieten, nicht als Unternehmerlnnen im klassischen Sinn verstehen, sind daher über gängige Informationskanäle in der Innovations- und Technologieförderung (z. B. einschlägigen Zeitschriften und Newslettern) nur bedingt zu erreichen und gehören auch nicht zur klassischen Klientel der Trägerorganisationen von iP.

Mit Hilfe einer breit angelegten PR Kampagne wurde in der Folge versucht, eine möglichst hohe Zahl an Kreativwirtschaftsunternehmen zu erreichen. Ein besonderer Fokus lag auf dem Design der Vermarktungsinstrumente, um den hierbei kreativen Anspruch des Förderprogramms zu unterstreichen und den Brückenschlag zur Wirtschaftsförderung zu erleichtern. Neben entsprechenden Printmaterialien (Foldern etc.) und der iP Homepage spielten vor allem Roadshows, halbtägige Veranstaltungen zum Thema iP Förderung, als Marketinginstrumente eine bedeutende Rolle. So fanden im gesamten Bundesgebiet alleine im Zeitraum zwischen 30. November 2004 und 20. Jänner 2005 insgesamt sieben Roadshows statt, die allesamt regen Zuspruch fanden. ${ }^{4}$

Grafik 1 zeigt, wie die letztlich am 1. Call von iP teilnehmenden Betriebe und jene Personen, die sich auf der Homepage von iP registrieren ließen, vom Förderprogramm erfahren haben. Bedeutendste Informationsquelle ist demnach das Internet bzw. die iP Homepage, über die etwa $39 \%$ der InteressentInnen und EinreicherInnen von der Initiative Kenntnis erlangten.

$27 \%$ gaben die Wirtschaftskammer als erste Informationsquelle an. Dieser vergleichsweise hohe Anteil zeigt, dass es der arge creativwirtschaft der Wirtschaftskammer Österreich gelungen ist, eine signifikante Zahl an Betrieben für iP zu mobilisieren. Unter der Berücksichtigung der vorher erwähnten Reserviertheit vieler Kreativer gegenüber dem Wirtschaftssektor wäre allerdings zu hinterfragen, inwieweit die avisierte Zielgruppe tatsächlich erreicht wurde. So bestehen Hinweise darauf, dass teilweise Betriebe angesprochen wurden, die nur bedingt der Kreativwirtschaft zuzurechnen sind und iP eher als ein zusätzliches F\&E- bzw. Innovationsförderprogramm für technische Produktentwicklungen ansehen. Hierfür spricht einerseits der vergleichsweise hohe Anteil an Unternehmen im 1. Call von iP mit Erfahrung mit F\&E- und Innovationsförderprogrammen (siehe Abschnitt 4.2.2) und auch die Tatsache, dass seitens der Juroren vielfach ein Mangel ein zu geringer kreativ-künstlerischer Gehalt in den Anträgen kritisiert wurde (siehe Abschnitt 4.2.1).

\footnotetext{
${ }^{4}$ Vgl. ARGE iP, Doku 1. Teil, 2005.
} 
$25 \%$ der Unternehmen haben über persönliche Kontakte in der Creative Industries Community von iP erfahren, was die Bedeutung der informellen Kommunikation und entsprechender Multiplikatoreffekte für die Unternehmensansprache von iP unterstreicht. In diesem Licht ist auch der vergleichsweise geringe Anteil von $5 \%$ an Betrieben zu sehen, der über die Roadshows von iP Kenntnis erlangte. Kommentare der Unternehmen und auch die ExpertInneninterviews deuten darauf hin, dass die Roadshows speziell wegen der Multiplikatorwirkungen als vorteilhaftes Marketinginstrument angesehen werden können.

Grafik 1 Informationskanäle, über die die Unternehmen vom iP ImpulsProgramm creativwirtschaft erfahren haben, Betriebe in Prozent *)

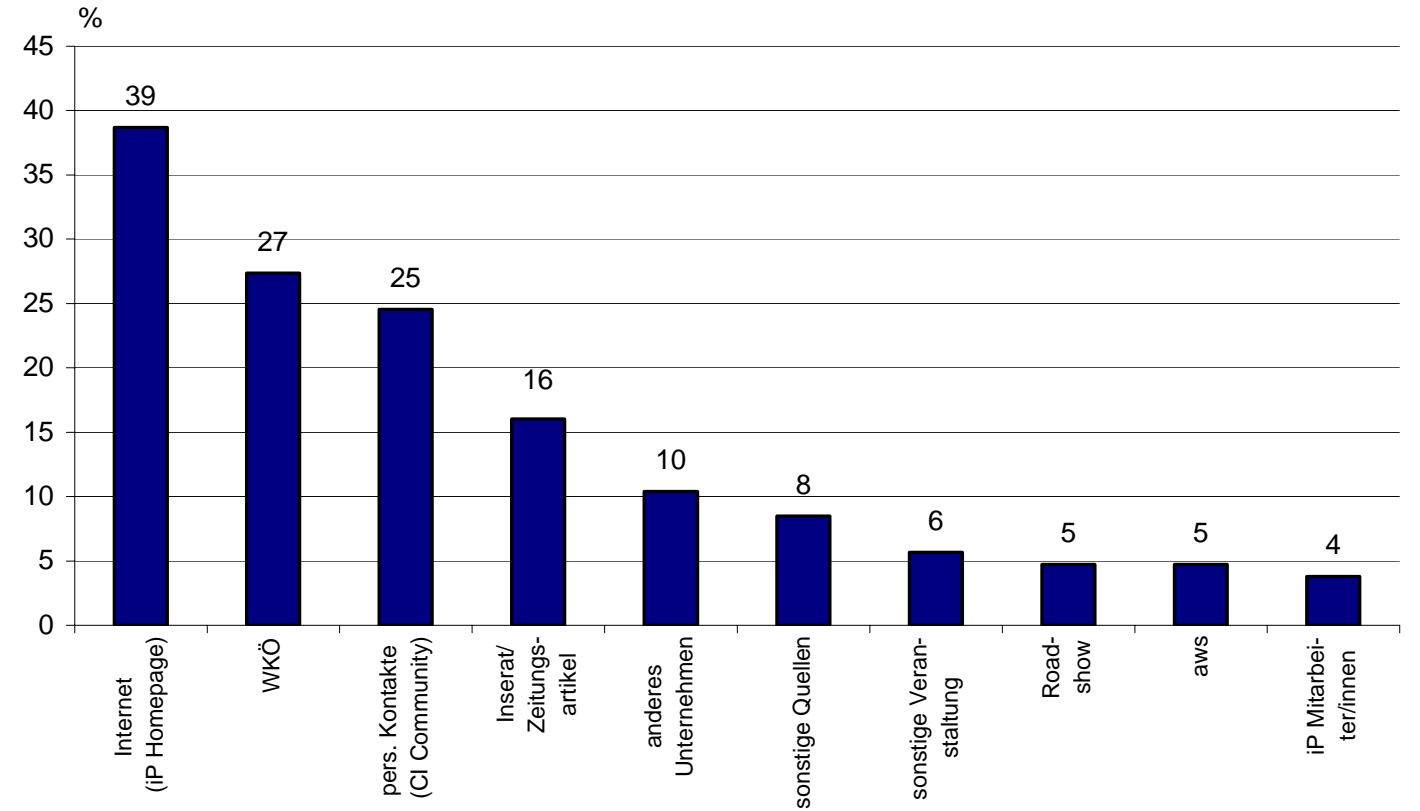

*) Mehrfachantworten möglich

Quelle: KMU FORSCHUNG AUSTRIA, N = 106

Sonstige Verbreitungskanäle spielten, zumindest für den 1. Call von iP, für die Unternehmensansprache eine geringere Bedeutung - so z. B. Inserate und Zeitungsartikel, über die etwa $16 \%$ der Unternehmen erreicht wurden, oder andere Unternehmen, durch die lediglich $8 \%$ auf iP aufmerksam wurden.

Etwa $49 \%$ der Unternehmen gaben an, dass iP in der Creative Industries Community ihrer Einschätzung nach „bekannt“ oder „sehr bekannt“ sei (siehe Grafik 2), wobei hinsichtlich dieser Einschätzung kaum Unterschiede zwischen den verschiedenen thematischen Bereichen und den Phasen der Antragsstellung auszumachen waren. Selbst unter den Einschränkungen, dass die Sichtweise der an iP teilnehmenden Unternehmen eine Befragung einer von iP unabhängigen Zufallsstichprobe an Unternehmen in der Kreativwirtschaft nicht ersetzen kann und dass, wie mehrfach ausgeführt, die von iP avisierte Zielgruppe nur zum Teil erreicht wurde, ist dieses Resultat - angesichts der geringen Zeit, die zur Umsetzung der PR Strategie im Vorfeld des 1. Calls zur Verfügung stand - positiv zu bewerten. 


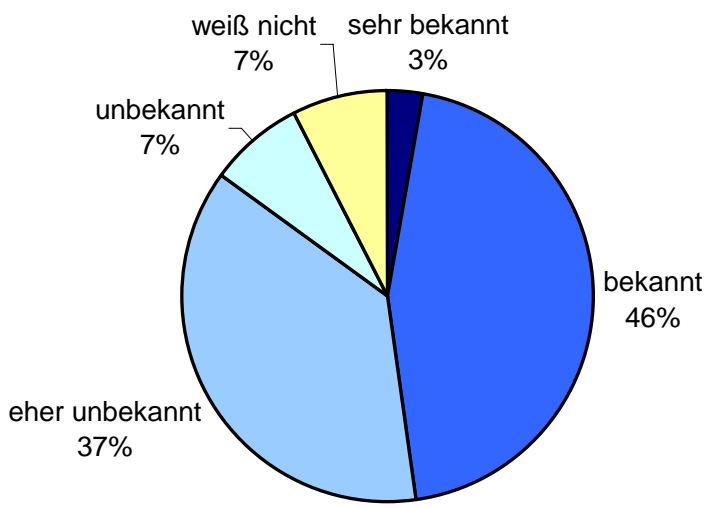

Quelle: KMU FORSCHUNG AUSTRIA, N = 107.

Im Rahmen der Evaluierung war des Weiteren zu klären, inwieweit die Trägerorganisationen der Initiative selbst und ihre Rolle im Zusammenhang mit iP von den einreichenden Unternehmen wahrgenommen wurden (siehe Grafik 3). Es zeigt sich, dass jeweils etwa 4 von 10 Betrieben die korrekten Funktionen der arge creativwirtschaft der WKO und der aws wiedergeben konnten, wobei die Unternehmen der arge creativwirtschaft (wohl als Folge des weiter oben angeführten hohen Mobilisierungsgrades durch die WKO) insgesamt etwas häufiger die richtigen Rollen zuordneten als der aws. Allerdings gaben auch $35 \%$ (bei der arge creativwirtschaft) bzw. $29 \%$ (bei der aws) an, dass die Rollen nach außen hin nicht klar genug vermittelt wurden. Etwa ein Fünftel kannte die genaue Rolle der aws nicht, selbiges traf zudem auf $29 \%$ hinsichtlich der arge creativwirtschaft zu.

Unterschiede beim Kenntnisstand bestehen bei Differenzierung nach Antragsphasen: Die Kenntnis der korrekten Funktion/Rolle der aws ist unter den Förderempfängern deutlich höher ausgeprägt als bei den Unternehmen in der Langantragsphase, während für die arge creativwirtschaft das Gegenteil gilt. Erwartungsgemäß war in der Kurzantragsphase (wo man sich noch am vergleichsweise wenigsten mit Details zu iP beschäftigt hat) der Kenntnistand sowohl hinsichtlich der aws als auch der arge creativwirtschaft am geringsten. Insgesamt dürfte jedoch hinreichend Wissen über die Trägerorganisationen und ihren Aufgaben im Rahmen von iP bei den einreichenden Betrieben vorhanden sein. Dies gilt vor allem vor dem Hintergrund des Bestrebens, iP als eigenständige Marke zu etablieren, aber auch auf Grund der Tatsache, dass die Kenntnis der Rollen der Trägerorganisation für die Antragserstellung von eher untergeordneter Bedeutung ist. 


\section{Grafik 3}

Wahrnehmung der Rollen der arge creativwirtschaft und der aws hinsichtlich iP *)

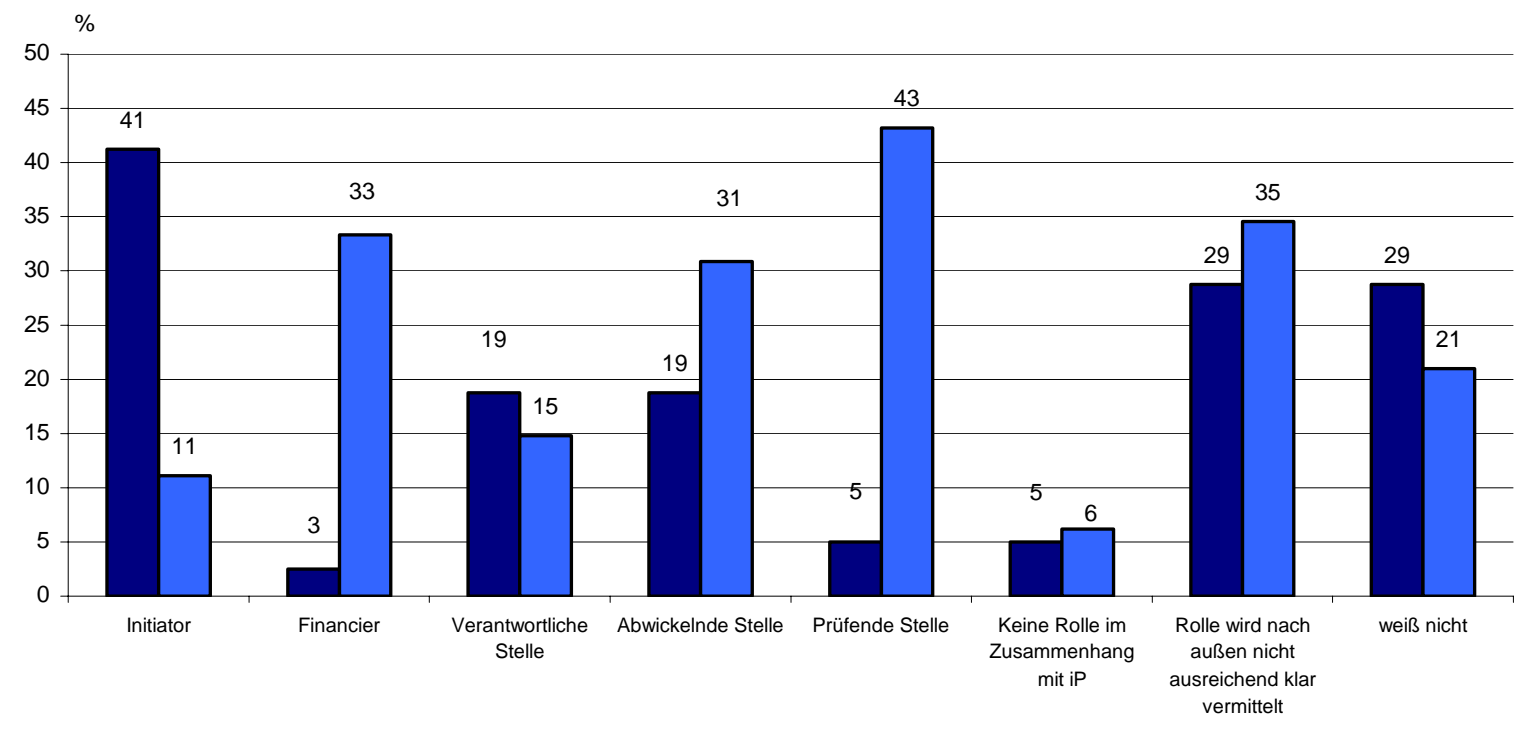

arge creativwirtschaft $\square$ aws

*) Mehrfachantworten möglich

Quelle: KMU FORSCHUNG AUSTRIA, N = 80.

Grafik 4 stellt die Zufriedenheit der Unternehmen (Einreicher und Interessenten) mit verschiedenen Aspekten des generellen Programmmanagements dar. Die durchschnittlichen Bewertungen - auf einer vierstufigen Skala von 1=sehr zufrieden bis 4=gar nicht zufrieden - bewegen sich in einer engen Bandbreite zwischen 1,9 (Erreichbarkeit der iP Mitarbeiterlnnen) und 2,2 (Organisation anderer Veranstaltungen als Roadshows, Klarheit der Informationsunterlagen). Dies kann als positives Resultat bezeichnet werden.

\section{Grafik 4 Zufriedenheit mit Aspekten des generellen Programmmanagements *)}

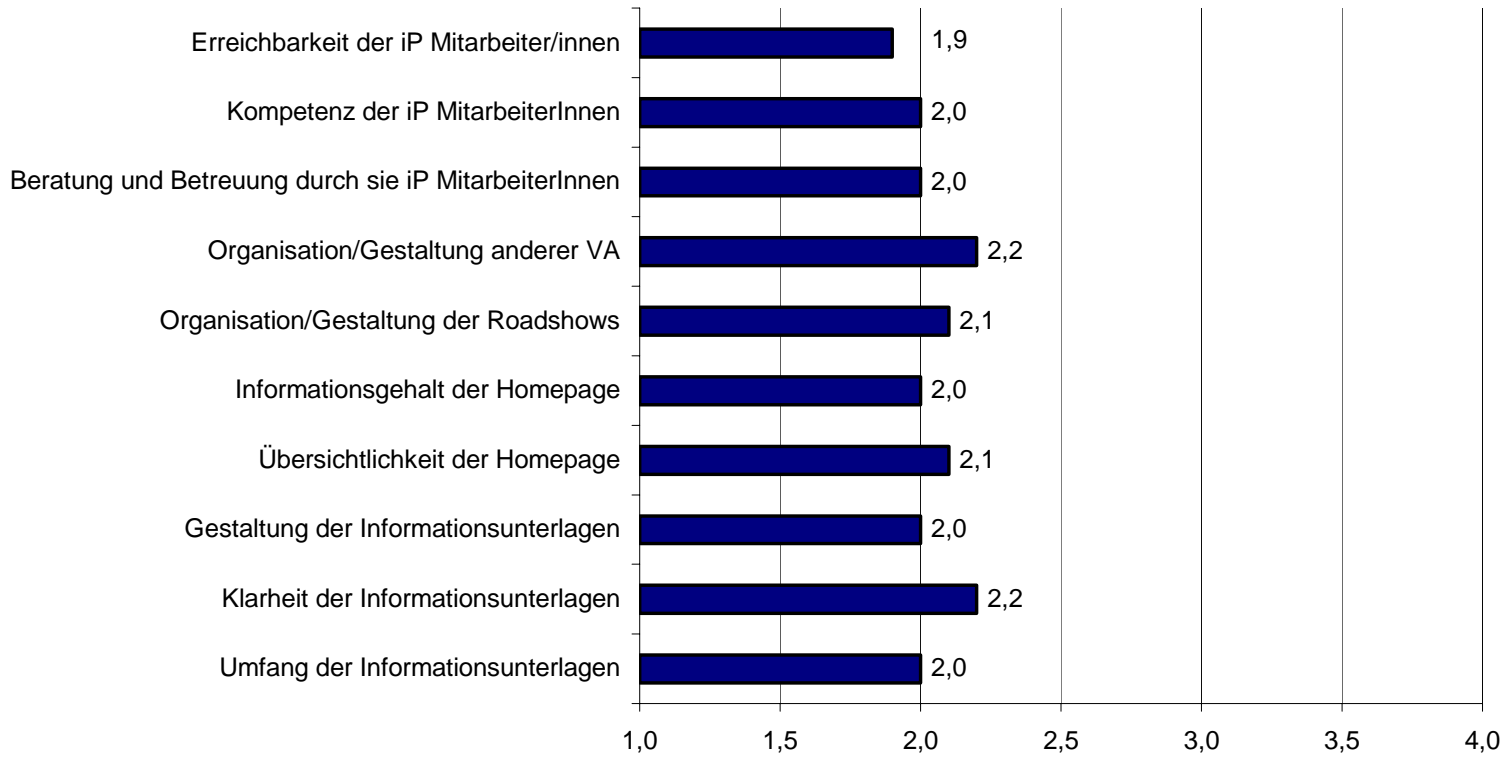

*) arithmetische Mittelwerte auf einer Skala von 1 = sehr zufrieden, 2 = zufrieden, 3= weniger zufrieden, 4= gar nicht zufrieden

Quelle: KMU FORSCHUNG AUSTRIA, N = 88. 
Leichte Unterschiede bestehen bei Differenzierung nach Fachbereichen (siehe Grafik 5): Unternehmen aus dem Bereich Multimedia scheinen mit dem generellen Programmmanagement meist etwas zufriedener zu sein als Betriebe in den Bereichen Design und Musik. Nichtsdestotrotz herrscht auch bei letztgenannten Themenbereichen ein relativ hohes Zufriedenheitsniveau vor.

Grafik 5 Zufriedenheit mit Aspekten des generellen Programmmanagements, nach Fachbereichen *)

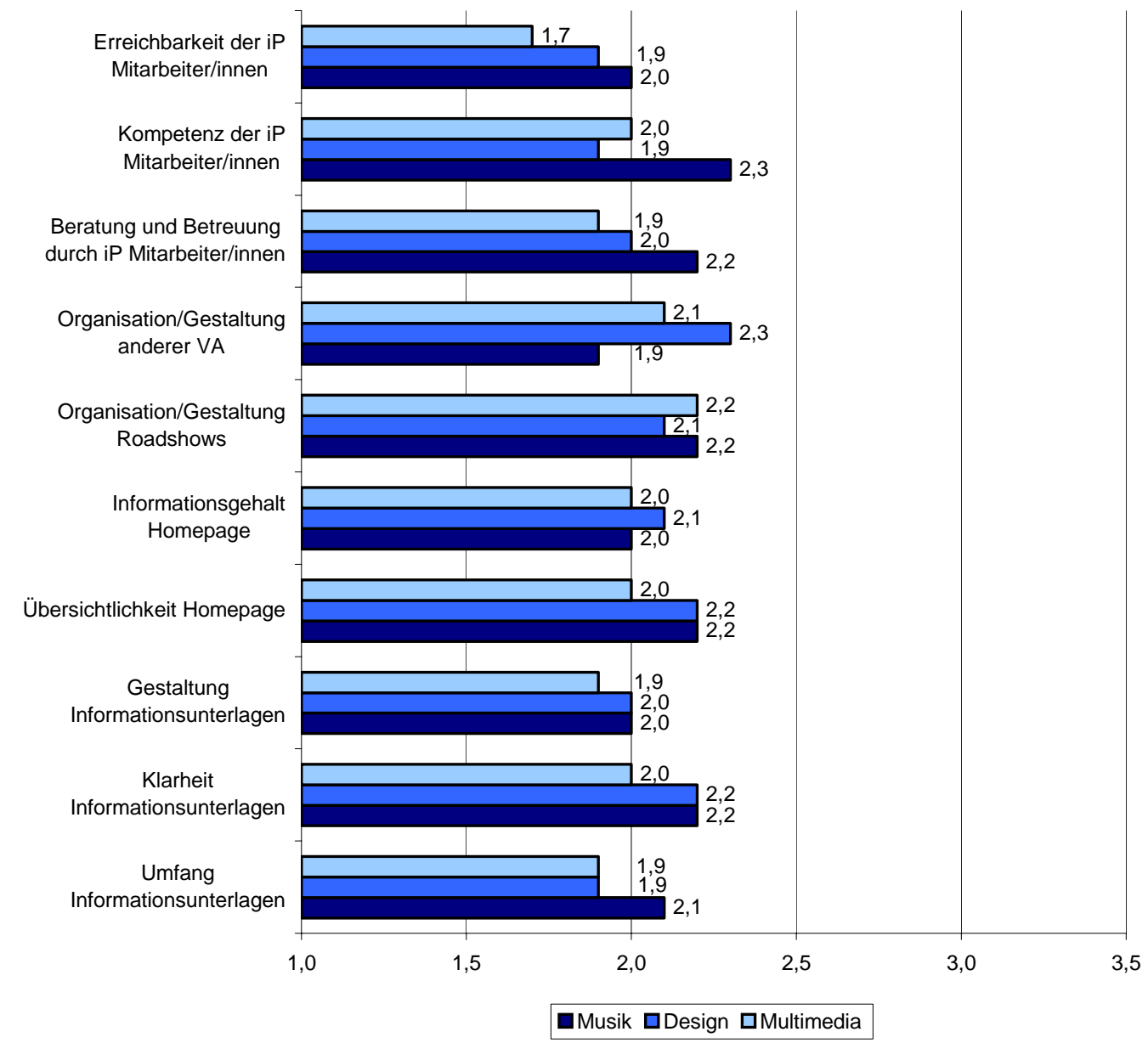

*) arithmetische Mittelwerte auf einer Skala von 1 = sehr zufrieden, 2 = zufrieden, $3=$ weniger zufrieden, $4=$ gar nicht zufrieden

Quelle: KMU FORSCHUNG AUSTRIA,

Die positiven Ergebnisse der standardisierten Unternehmensbefragung hinsichtlich des generellen Programmmanagements werden durch die Interviewresultate gestützt. So wird den PR Tätigkeiten ein gutes Zeugnis ausgestellt, wobei der Nutzen der Roadshows für Multiplikatoreffekte und die professionell gestalteten Drucksorten von vielen InterviewpartnerInnen hervorgehoben wurden. Auch der Einsatz und das Engagement des iP Büros fanden häufig lobend Erwähnung. Kritikpunkte bzw. Verbesserungsvorschläge gehen vor allem in die Richtung, in der Öffentlichkeitsarbeit vermehrt konkrete Programminhalte zu vermitteln. Damit könnte auch ein wichtiger Beitrag zur Verbesserung der Transparenz (siehe Abschnitt 4.2.3) geleistet werden. 


\subsection{Abwicklung des 1 . Calls von iP}

\subsubsection{Der Ablauf des 1. Calls des iP ImpulsProgramm creativwirtschaft}

Der 1. Call im Rahmen des iP Impulsprogramm creativwirtschaft fand im Zeitraum zwischen Dezember 2004 (Einreichung der Anträge: ab dem 15.12.2004) und Juni 2005 (Endtermin für die Bekanntgabe der Förderzusagen: bis spätestens 27.6.2005) statt. Da die Entscheidung zur Durchführung von iP erst im Sommer 2004 gefallen ist, und die erste grundsätzliche Programmkonzeption auch erst im Frühjahr 2004 stattgefunden hat, stand für die Vorbereitung des 1. Calls (angesichts dessen, dass auch die gesamte Infrastruktur aufgebaut werden musste) vergleichsweise wenig Zeit zur Verfügung. Der zweite Call findet im Zeitraum Dezember 2005 bis Juni 2006 statt.

Für die Durchführung der Calls wurde ein zweistufiges Verfahren gewählt. In der ersten Phase haben die Einreicher nur eine Kurzversion ihres Antrages mit wichtigen Kenndaten zum Unternehmen und zum Projekt einzubringen. Jene Betriebe, die die besten Kurzanträge geschrieben hatten, werden im Anschluss eingeladen, ihre Projektideen und ihren Businessplan in einem Langantrag detailliert auszuarbeiten.

Um am Call zugelassen zu werden, müssen die Betriebe bestimmte Basiskriterien erfüllen. So muss das Projekt zunächst einen Zusammenhang mit dem Entwurf, der Entwicklung, Herstellung, Anwendung oder Vermarktung von innovativen Produkten, Verfahren oder Dienstleistungen in den Themenbereichen Multimedia, Design und Musik aufweisen. Des Weiteren wird ein hinreichender Konkretisierungsgrad gefordert. Schließlich sind auch die wirtschaftliche Umsetzbarkeit und die Marktorientierung der Vorhaben darzulegen. Explizit ausgeschlossen werden z. B. Projekte im Bereich der Grundlagenforschung oder Vorhaben, die routinemäßige Änderungen bestehender Produkte, Dienstleistungen und Verfahren zum Inhalt haben.

Die aus den Langanträgen ausgewählten Förderempfänger erhalten einen finanziellen Zuschuss für die Abwicklung der Projekte, die so genannte iP Förderung. Finanziert werden maximal $40 \%$ der Kosten für materielle und immaterielle Investitionen im Zusammenhang mit den beantragten Projekten und bis zu $70 \%$ der sonstigen Projektkosten. Die iP Förderung ist als "de-minimis" Förderung ausgelegt, weswegen die Maximalförderhöhe $€ 100.000$,- innerhalb von drei Jahren nicht übersteigen darf. Die Auszahlungsmodalitäten sehen die Zahlung von $30 \%$ der Fördersumme bei Projektstart, von $40 \%$ bei Nachweis des Verbrauchs von $50 \%$ der Projektkosten und Erstellung eines Zwischenberichts und der restlichen $30 \%$ bei Projektabschluss und Vorlage eines Endberichtes vor. Der Zwischen- und der Endbericht waren als Meilensteine in den Anträgen vorzugeben und dienen auch dem Projektmonitoring.

Insgesamt wurden 315 Projekte bzw. Kurzanträge im Rahmen des 1.Calls von iP eingereicht, wobei der Großteil der Projekte auf den Bereich Multimedia (123 Projekte) entfiel, gefolgt von den Bereichen Design (109 Projekte) und Musik (83 Projekte) (siehe Tabelle 2). Damit lag die Zahl der Anträge weit über den Erwartungen des iP Programmmanagements und übertraf Experten zufolge - auch ähnlich gelagerte Förderprogramme in Deutschland.

51 der 315 Projekte (etwa ein Fünftel) wurden für die Langantragsphase ausgewählt. 24 Projekte wurden schlussendlich gefördert, davon 11 im Bereich Multimedia, acht Designprojekte und fünf Musikprojekte. Dies entspricht einer Erfolgsquote (Anzahl der Förderempfänger im Verhältnis zur Summe der eingereichten Projekte aus Phase 1) von etwa 7,5\%. Die Erfolgsquote war im Bereich Multimedia (rd. $9 \%$ ) höher als im Design- (rd. $7 \%$ ) und Musikbereich (rd. $6 \%)$. 
Tabelle 2: $\quad$ Zahl der Projektanträge in den einzelnen Phasen des 1.Calls von iP

\begin{tabular}{l|c|c|c}
\hline & $\begin{array}{c}\text { Kurzantragsphase } \\
\text { (Phase 1) }\end{array}$ & $\begin{array}{c}\text { Langantragsphase } \\
\text { (Phase 2) }\end{array}$ & Förderempfänger \\
\hline Multimedia & 123 & 22 & 11 \\
Design & 109 & 17 & 8 \\
Musik & 83 & 12 & 5 \\
\hline \hline Gesamt & $\mathbf{3 1 5}$ & $\mathbf{5 1}$ & $\mathbf{2 4}$ \\
\hline \multicolumn{2}{l}{} \\
\hline
\end{tabular}

Deutliche Unterschiede waren nach Ansicht der JurorInnen bei der Qualität der Anträge auszumachen. Am relativ besten schnitten die Projekte im Bereich Multimedia ab, wo vor allem den letztlich geförderten Vorhaben hinsichtlich des Kreativitätsanspruches, des Innovationsgrades und auch der internationalen Wettbewerbsfähigkeit ein gutes Zeugnis ausgestellt wurde. Demgegenüber wiesen die Anträge im Bereich Design und noch mehr im Bereich Musik Schwächen auf. Vielfach konnten die Marktaussichten nicht plausibel dargestellt werden (z. B., weil Wettbewerbsanalysen fehlten), die wirtschaftliche Verwertbarkeit der Ideen war nicht unmittelbar nachzuvollziehen und/oder die Aufbereitung betriebswirtschaftlicher Daten erwies sich als ungenügend.

Experten zufolge spiegelt dieses Resultat aber auch den unterschiedlichen Kenntnisstand im Bereich der Unternehmensführung in den drei Bereichen wider. Erfahrungen in Deutschland mit Kreativwirtschaftsförderprogrammen zeigen, dass mit der Durchführung der Anzahl der durchgeführten Calls auch Lerneffekte bei den Betrieben zu beobachten sind und die Qualität der Projektanträge sukzessive steigt. Nichtsdestotrotz könnten zur Steigerung der Antragsqualität verschiedene Maßnahmen überlegt werden. Eine Möglichkeit wäre, je nach Themenbereich unterschiedliche bzw. unterschiedlich hohe Standards der Programmbewertung zu Grunde zu legen. Ebenso könnten Sondercalls überlegt werden, wo - unabhängig vom Themenfeld - der Kreativitätsaspekt bzw. die Ideen an sich noch stärker im Blickfeld stehen. Hier gilt es jedoch darauf zu achten, dass trotzdem ein gewisser Mindeststandard beibehalten wird, da sonst der administrative Aufwand für die Bearbeitung der Förderanträge den potenziellen Nutzen übersteigen würde. Als Maßnahme denkbar ist im jeden Fall auch ein Ausbau der Aus- und Weiterbildungsschiene im Bereich der Antragsberatung (siehe hierzu auch Abschnitt 4.3.2).

Neben den betriebswirtschaftlichen-marktorientierten Antragsteilen war das in Abschnitt 3.1 beschriebene Spannungsfeld zwischen Kreativitäts- und Innovationsförderung häufig ein Thema bei der Bewertung der Projektanträge. Zum Einen gaben einige Juroren an, dass der zu berücksichtigende Stellenwert des kreativ-künstlerischen Gehalts im Antrag im Verhältnis zum Innovationsgrad der Projekte von iP nicht ausreichend klar durch das iP Team vermittelt wurde. Zum Anderen wurde zumeist auch Enttäuschung darüber zum Ausdruck gebracht, dass in vielen Fällen kreative Elemente in den Anträgen und/oder ein eindeutiger Bezug zur Kreativwirtschaft gefehlt haben. Die in Abschnitt 2.1 angerissenen möglichen Konsequenzen von Projekten, denen ein klarer Konnex zur Kreativwirtschaft fehlt, lassen Präzisierungen in dieser Hinsicht notwenig erscheinen, vor allem vor dem Hintergrund einer möglichst hohen Transparenz (siehe auch Abschnitt 4.2.3). Die Kommentare der Experten weisen schließlich darauf hin, dass eine stärkere Betonung des Kreativanteils in den Anträgen das Standing und die Funktion von iP als Impulsgeber in den Creative Industries weiter heben bzw. verbessern könnten. 


\subsubsection{Struktur der einreichenden Unternehmen}

Jene Unternehmen, die im Rahmen des 1. Calls des iP ImpulsProgramm creativwirtschaft einen Antrag gestellt haben, sind überwiegend kleinbetrieblich strukturiert (siehe Grafik 6). Bei ca. $29 \%$ handelt es sich um Einpersonenunternehmen (EPU) ohne unselbstständig Beschäftigte. Nur $11 \%$ haben mehr als 5 MitarbeiterInnen. Der überwiegende Teil (60\%) sind Betriebe, die zwischen 1 und 5 MitarbeiterInnen beschäftigen. In dieser Verteilung spiegelt sich die Struktur der Kreativwirtschaft wider. ${ }^{5}$

Grafik 6 Großenverteilung der Unternehmen, die im Rahmen des 1. Calls von iP einen Antrag gestellt haben

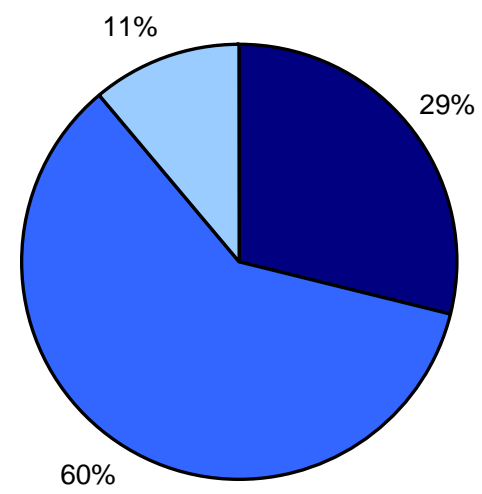

$\square E P U \square 1$ bis 5 Beschäftigte $\square$ mehr als 5 Beschäftigte

Quelle: KMU FORSCHUNG AUSTRIA, N = 80.

Die kleinstbetriebliche Struktur ist insbesondere im Bereich Musik anzutreffen, wo jedes einreichende Unternehmen im Durchschnitt eine einzige Person beschäftigt und kein einziges über mehr als fünf Beschäftigte verfügt. Zwischen den verschiedenen Antragsphasen variiert die dargestellte Größenverteilung hingegen nur geringfügig.

\footnotetext{
${ }^{5}$ Vgl. Erster Österreichischer Kreativwirtschaftsbericht, 2003, S. 31.
} 
Tabelle 3: Regionale Verteilung der Projektanträge nach Kurzantragsphase und Förderempfänger

\begin{tabular}{l|c|c|c|c}
\hline \multirow{2}{*}{} & \multicolumn{2}{|c|}{ Kurzantragsphase (Phase 1) } & \multicolumn{2}{c}{ Förderempfänger } \\
\cline { 2 - 5 } & Anzahl Anträge & Anteil in \% & Anzahl Anträge & Anteil in \% \\
\hline Wien & 124 & 39 & 13 & 54 \\
Steiermark & 63 & 20 & 3 & 13 \\
Oberösterreich & 31 & 10 & 0 & 0 \\
Salzburg & 25 & 8 & 2 & 8 \\
Niederösterreich & 24 & 8 & 0 & 0 \\
Vorarlberg & 22 & 7 & 2 & 13 \\
Tirol & 16 & 5 & 3 & 0 \\
Burgenland & 4 & 1 & 0 & 0 \\
Kärnten & 4 & 1 & 0 & 4 \\
Ausland & 2 & 1 & 1 & $\mathbf{1 0 0}$ \\
\hline \hline
\end{tabular}

Aus jedem Bundesland ist mindestens ein Projekt eingereicht worden (siehe Tabelle 3). Bei der regionalen Verteilung der letztlich geförderten Projekte blieb Wien mit einem Anteil von $54 \%$ an der Spitze. Unter den Bundesländern waren vor allem die Steiermark und Tirol mit jeweils 3 Anträgen am vergleichsweise stärksten vertreten.

Etwa $48 \%$ der Unternehmen wurden nach 2003, $21 \%$ im Zeitraum 2000 bis 2002 und $31 \%$ vor dem Jahr 2000 gegründet (siehe Grafik 7). Nur im Musikbereich, der sich auf Grund der vergleichsweise geringen Anzahl an Einreichungen weniger auf das Gesamtergebnis niederschlägt, unterscheidet sich die Verteilung der Anteilswerte deutlich: Hier ist der Anteil der Gründungen nach 2003 niedriger und liegt bei rd. $29 \%$.

Grafik 7 Gründungsjahr der Unternehmen, die im Rahmen des 1. Calls von iP einen Antrag gestellt haben

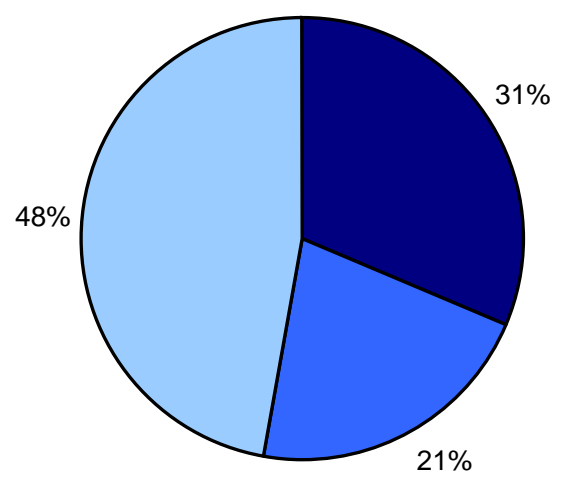

bis 1999 口2000 bis 2002 प2003 und jünger

Quelle: KMU FORSCHUNG AUSTRIA 
Etwa $28 \%$ der Betriebe wurden anlässlich der Teilnahme am iP ImpulsProgramm creativwirtschaft gegründet. Differenziert nach Antragsphasen zeigt sich, dass in Phase 1 (Kurzantragsphase) der Anteil der speziell für iP gegründeten Betriebe mit rd. $34 \%$ deutlich über dem entsprechenden Anteil in späteren Antragsphasen lag (19\%). Dies dürfte eine höhere Erfahrung bestehender Unternehmen in betriebswirtschaftlichen Belangen und in der Antragsverfassung widerspiegeln. Unterschiede bestehen des Weiteren auch zwischen den einzelnen thematischen Bereichen. So lag der Anteil der auf iP zurückzuführenden Gründungen im Bereich Multimedia nur bei ca. 18 \%, während diese im Musikbereich (27\%) und vor allem im Designbereich (41\%) eine wesentlich prominentere Rolle innehatten.

Etwa $18 \%$ der Einreicher haben in den vergangenen fünf Jahren an Innovations- und F\&EFörderprogrammen teilgenommen, wobei es sich hier meist um Einzelprojektförderungen des Forschungsförderungsfonds (FFF, nunmehr der Bereich Basisprogramme in der Forschungsförderungsgesellschaft (FFG)) gehandelt hat. $16 \%$ haben Anträge im Rahmen von Kreativwirtschaftsprogrammen gestellt (hauptsächlich in Wien bei departure), $14 \%$ im Rahmen von Programmen der sonstigen Wirtschaftsförderung. Etwa $3 \%$ hat sich an Kunst- und Kulturförderprogrammen beteiligt oder in diesem Bereich einen Preis bzw. eine Auszeichnung erhalten. Insgesamt können etwa 40 \% der Unternehmen auf Erfahrungen mit anderen Förderprogrammen zurückgreifen.

Grafik 8 Teilnahme an anderen Förderprogrammen in den vergangenen fünf Jahren, Einreicher in Prozent *)

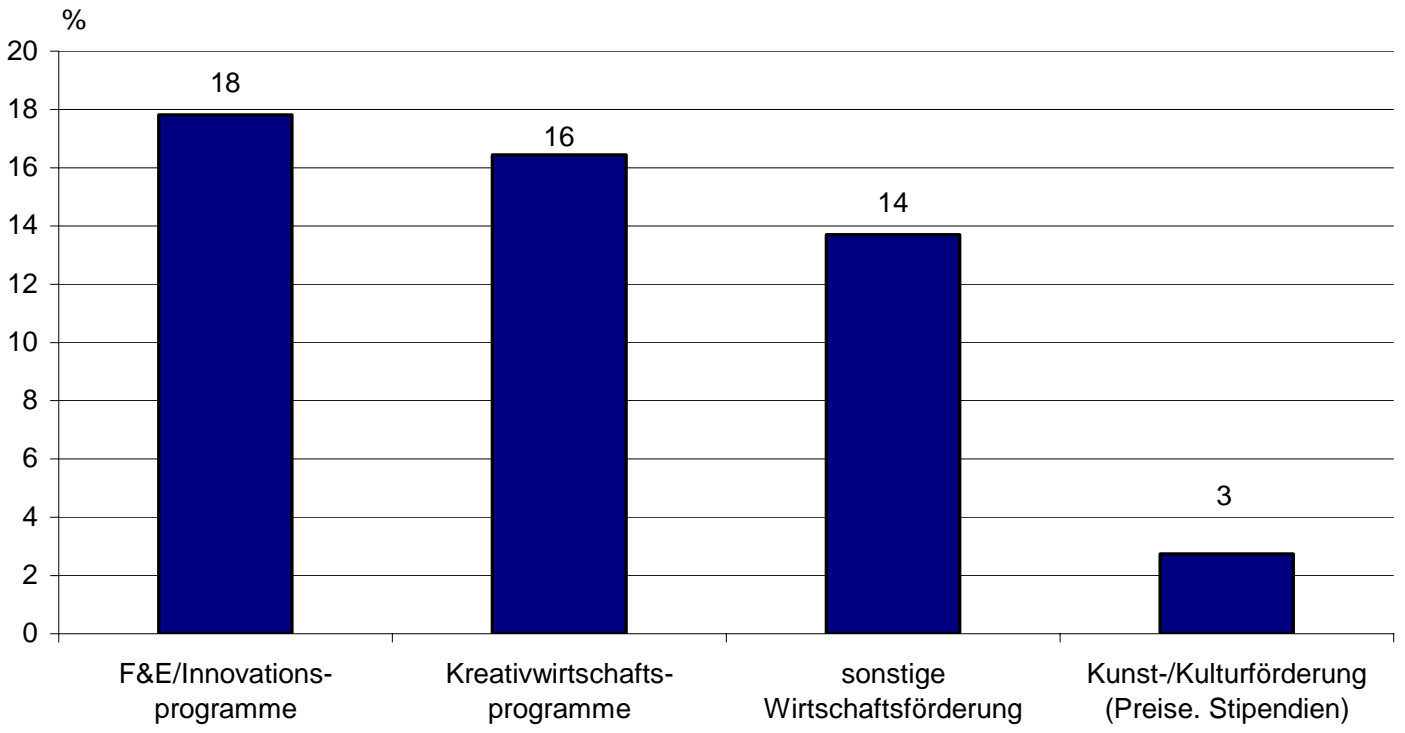

*) Mehrfachantworten möglich

Quelle: KMU FORSCHUNG AUSTRIA, N = 73

Die Ergebnisse legen nahe, dass iP eine breite Palette an bestehenden (mehrheitlich aber jungen) und neuen, anlässlich des 1. Calls gegründeten Unternehmen erreicht und gefördert hat. Dies ist - auch im Vergleich zu entsprechenden Programmen in Deutschland, die hauptsächlich im Gründungsbereich aktiv sind - zu begrüßen, da damit die Initiative seiner Rolle als Impulsbringer in der Kreativwirtschaft gerecht wird, gleichzeitig von den Erfahrungen bestehender Betriebe profitiert und darüber hinaus keine bestehenden Förderprogramme zu Unternehmensgründungen dupliziert.

Der überraschend hohe Anteil an Betrieben, die über Erfahrungen mit Förderprogrammen, allerdings nicht aus dem Kunst- und Kulturbereich, verfügen, ist ein (weiterer) Hinweis darauf, dass die avisierte Zielgruppe der Kreativen nur teilweise erreicht wurde. Viele Betriebe dürften iP nur als alternatives Innovationsförderprogramm ansehen und ansonsten nur geringen Bezug zur Kreativwirtschaftsszene haben. 


\section{Charakteristika der geförderten Projekte}

Die 24 geförderten Projekte weisen ein Gesamtprojektvolumen von $€$ 5,8 Mio auf, wovon auf die Bereiche Multimedia und Design $€ 2,7$ Mio bzw. $€ 2,3$ Mio entfallen. Der Bereich Musik hat ein Gesamtprojektvolumen von $€ 800.000$,-. Die durchschnittliche Fördersumme pro Projekt liegt im Bereich Multimedia bei rd. €93.000,-, im Bereich Design bei ca. €95.000,- und im Bereich Musik bei etwa $€ 86.000,-$.

Fünf Betriebe gaben dezidiert an, dass sie für ihre Projekte Kofinanzierungen beziehen, acht weitere verneinten dies. Zur Verfügung gestellt wurden die Mittel von der Stadt Wien (in zwei Fällen), dem Land Niederösterreich und dem Land Vorarlberg.

Bezüglich der Projektorganisation ist noch anzumerken, dass etwas weniger als ein Viertel der Projekte von einer Frau geleitet wird.

\subsubsection{Das Projektauswahlverfahren}

Die Bewertung der Anträge im zweistufigen Callverfahren erfolgte einerseits durch Jurys, die den inhaltlichen Teil der Anträge zu beurteilen hatten, und andererseits durch die aws, die für die Analyse der betriebswirtschaftlichen Teile zuständig war. Für jeden der drei Themenbereiche wurde eine eigene Jury gebildet, die aus sieben (Design) bzw. acht (Musik, Multimedia) entsprechenden ExpertInnen bestand. Zwei (Design) bzw. drei (Musik und Multimedia) der JurorInnen stammten hierbei aus dem deutschsprachigen Ausland, die restlichen MitgliederInnen aus Österreich.

Die JurorInnen hatten die Anträge an Hand von sieben Kriterien zu bewerten:

1. Innovationsgrad (einschließlich des künstlerisch-kreativen Gehaltes) der Projekte

2. Wirtschaftliche Umsetzbarkeit und Marktorientierung

3. Kooperationen und/oder Clusterbildung zwischen bzw. unter KMU, F\&E-Einrichtungen und Ausbildungsinstitutionen

4. Unternehmensgründungen

5. Nachhaltige Auswirkungen des Projektes auf die Unternehmensentwicklung

6. Finanzieller Beitrag und Risikoanteil des KMU

7. Beitrag des Projektes zur Wertschöpfungskette (z. B. Schaffung von Arbeitsplätzen) in Österreich

Im Vorfeld der Jurysitzungen wurden die Anträge in betriebswirtschaftlicher Hinsicht von der aws mit Hilfe eines quantitativen Punkte-Wertungssystems geprüft. Das angewendete Beurteilungsschema entsprach jenem, das die aws auch in ihren anderen Förderprogrammen anwendet. Aus der Bewertung der einzelnen Kriterien ergab sich hierbei für jedes Projekt eine Gesamtpunkteanzahl, die den Jurorlnnen für die weitergehende Beurteilung des inhaltlichen Teils mitgeteilt wurde. Im weiteren Verlauf war die aws auch in den Jurysitzungen aller drei Bereiche (Design, Multimedia und Musik) als „wirtschaftlicher Experte“ präsent.

Alle JurorInnen erhielten individuelle Zugangsdaten zum elektronischen Einreichsystem von iP, mit denen es möglich war, die Anträge online zu betrachten. Auch die Abgabe der Wertungen (Begründungen samt Vergabe von „Noten“ nach einem Punkteschlüssel) erfolgte auf elektronischem Wege. Die einzelnen Projektbewertungen bildeten schließlich die Diskussionsbasis für zwei jeweils eintägige Jurysitzungen. In diesen Sitzungen wurde dann eine Reihung der Projekte für die Zulassung zur Langantragsphase (bzw. in der zweiten Sitzung für die endgültige Förderzusage) vorgenommen. 
Der Ablauf der Bewertungsprozesses, wie auch die Zusammensetzung der und die Zusammenarbeit in den Jurys, wurde von den JurorInnen prinzipiell als zufrieden stellend empfunden. Speziell die Wahl des zweistufigen Verfahrens wurde als sinnvoll angesehen. Lobende Erwähnung fand der Einsatz des iP Büros und die Arbeit der aws bei der betriebswirtschaftlichen Bewertung. Kritikpunkte bestanden lediglich in Detailbereichen:

- Der Aufwand zur Bearbeitung der Anträge wurde zum Teil als sehr hoch empfunden, was aber auch durch die unerwartet hohe Zahl der Anträge begründet war.

- Die Einbeziehung der internationalen Experten erfolgte erst in der Langantragsphase. Diese äußerten jedoch den Wunsch, bereits in der Vorselektion in Phase I mitwirken zu können (was sicher als sinnvoll anzusehen ist).

- Das speziell für iP entwickelte elektronische Einreichsystem hat zwar großteils zuverlässig funktioniert, wies aber funktionelle Einschränkungen auf. So gab es z. B. keine Möglichkeit, die Anträge als ganze zusammenhängende Dateien auszudrucken. Speziell im Designbereich wurde die fehlende Möglichkeit, Bilder der Projekte betrachten zu können, kritisiert.

- Die Tatsache, dass die Jurysitzungen in den drei Themenbereichen parallel stattgefunden haben, hat sich im Hinblick auf eine Beteiligung/Beobachtung durch das iP Programmmanagement als problematisch herausgestellt.

- Mehrfach wurde gefordert, noch stärker als bisher internationale Experten in den Jurys einzubinden

Die meisten dieser Problembereiche wurden auch bei der Bildung der Expertenbeiräte aufgegriffen und im 2. Call beseitigt. So gibt es beispielsweise ein neues elektronisches Einreichtool, internationale Experten sind bereits in der Kurzantragsphase tätig und Jurysitzungen werden nicht mehr parallel abgehalten. Im Bezug auf die höhere Internationalität könnte überlegt werden, inwieweit die Einbindung englischsprachiger Experten, speziell aus Großbritannien, sinnvoll ist. Hier wäre der Nutzen (ein evtl. besseres Standing des Programms, höhere Sichtbarkeit der Projekte im internationalen Kontext und vor allem eine möglicherweise nochmals erhöhte Transparenz) dem Aufwand (Antragserstellung auf Englisch, evtl. höhere Kosten für die Abgeltung der JurorInnentätigkeit) gegenüber zu stellen. Alternativ wäre auch eine Einbindung in den Expertenbeiräten vorstellbar.

Die Grafiken 9 und 10 stellen die Zufriedenheit der am 1. Call teilnehmenden Unternehmen mit verschiedenen Aspekten des Projektauswahlverfahrens dar. Hierbei zeigt sich, dass die Bewertung der Projektnehmer - im Vergleich zu jener des generellen Programmmanagements deutlich heterogener ausfällt. Erwartungsgemäß war die Zufriedenheit unter den Förderempfängern generell höher als in den anderen Antragsphasen (siehe Grafik 10). Analog bewerteten jene Betriebe, die einen Langantrag gestellt und letztlich nicht gefördert wurden, die Callabwicklung am relativ schlechtesten.

Positiv beurteilt wurden - in allen Antragsphasen - die Zeit, die für die Antragserstellung zur Verfügung stand (im Durchschnitt mit „sehr zufrieden“ bis „zufrieden“), die Zuverlässigkeit des elektronischen Einreichsystems (Durchschnittsnote je nach Antragsphase zwischen 1,8 und 2,1 auf einer vierstelligen Skala von $1=$ sehr zufrieden bis $4=$ gar nicht zufrieden) und die Bearbeitungsdauer von Anfragen zum Projektantrag (Durchschnittsnote: 1,8 bis 2,2). Auch der weiteren Abwicklung der Förderung durch die aws nach Förderzusage wurde seitens der Projektnehmer ein gutes Zeugnis ausgestellt (durchschnittliche Bewertung: 2,1). 
Etwas schlechter als die Zuverlässigkeit des elektronischen Einreichsystems wurde dessen Benutzerfreundlichkeit und Funktionalität empfunden, speziell bei den Unternehmen in der Langantragsphase. Hier spielt, wie in Abschnitt 4.2.3 bereits dargestellt, hinein, dass Z. B. keine Möglichkeit bestand, Bilder zur Beschreibung der eigenen Projekte auf den iP Server aufzuspielen.

Grafik 9 Zufriedenheit mit verschiedenen Aspekten des Projektauswahlverfahrens, arithmetische Mittelwerte *), Einreicher gesamt

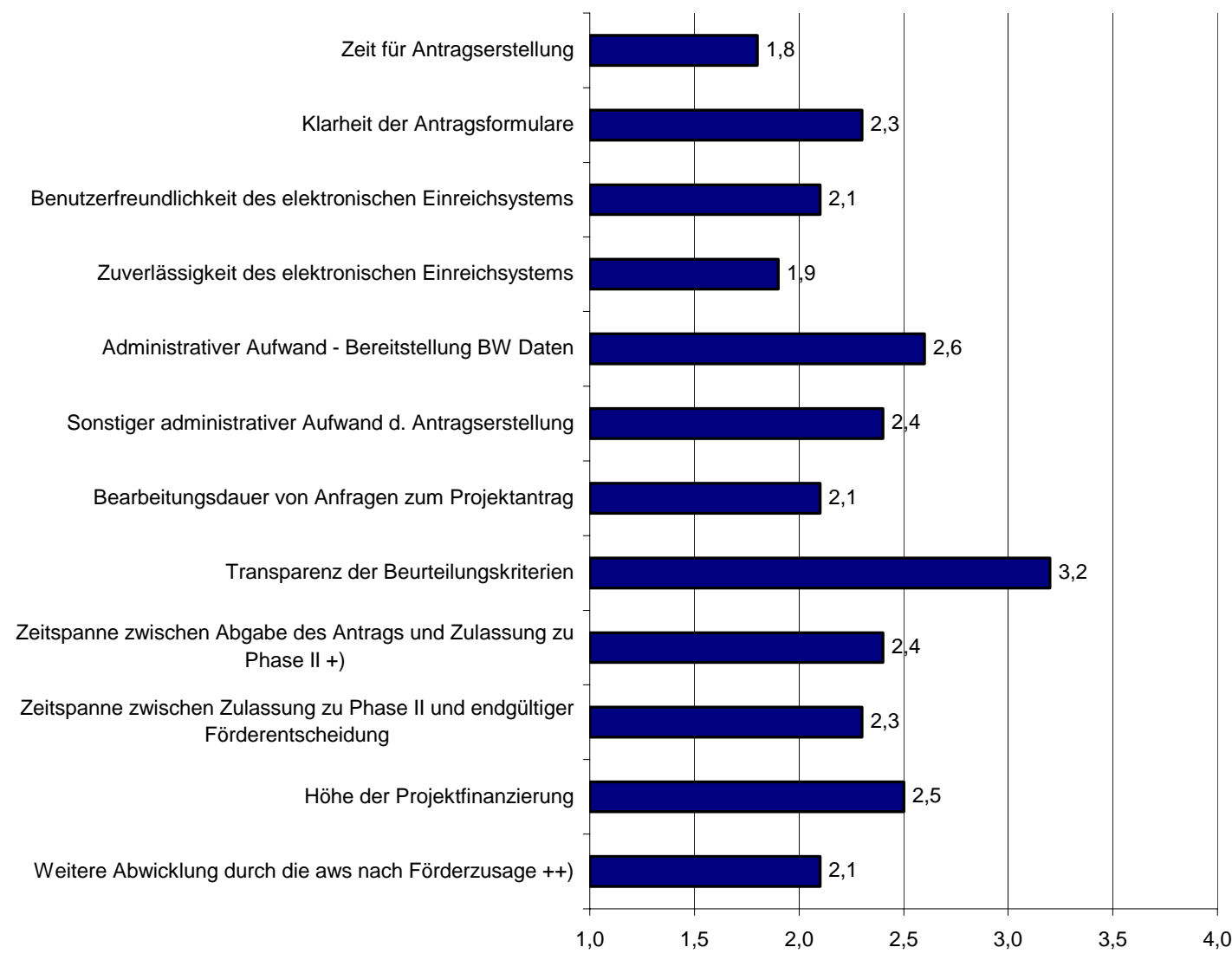

+) Diese Frage wurde den Unternehmen in der Kurzantragsphase nicht gestellt.

++ ) Diese Frage wurde Unternehmen in der Kurz- und in der Langantragsphase nicht gestellt.

*) auf einer Skala von 1 = sehr zufrieden, 2 = zufrieden, 3 = weniger zufrieden, 4 = gar nicht zufrieden

Quelle: KMU FORSCHUNG AUSTRIA, N = 81

Relativ heterogen wurden die Klarheit der Antragsunterlagen, die Zeitspanne zwischen Abgabe des Antrags und Zulassung zur Langantragsphase sowie die Zeit zwischen der Zulassung zur Phase II und der endgültigen Förderentscheidung bewertet. Dies gilt des Weiteren auch für die Höhe der Förderung (Durchschnittsbewertung: 2,5). Kommentare der Betriebe weisen allerdings darauf hin, dass sich hier eher der Wunsch nach einer umfassenderen Förderung als Kritik an der konkreten Programm- bzw. Callabwicklung widerspiegelt. 
In Bezug auf die Zeitaspekte dürfte zum Tragen kommen, dass viele Unternehmen den Aufwand für die Erstellung eines fundierten Proposals unterschätzt zu haben scheinen. In diesem Zusammenhang ist eventuell auch die schlechte Bewertung hinsichtlich des Aufwands zur Bereitstellung betriebswirtschaftlicher Daten (Durchschnittsnote: 2,6 unter allen Einreichern und 2,9 in der Langantragsphase) zu sehen. Der doch deutlich negative Bias bei diesem Aspekt könnte aber auch darauf hindeuten, dass die Anforderungen tatsächlich zu hoch gesteckt sind, speziell vor dem Hintergrund, dass viele Unternehmen gerade gegründet wurden. Entsprechend sollten Möglichkeiten zur Reduzierung des Umfangs der bereitzustellenden betriebswirtschaftlichen Daten geprüft werden, ohne dass dadurch die Qualität der Anträge beeinträchtigt wird. Sinnvoll erscheint des Weiteren ein Ausbau der Begleitmaßnahmen wie den Businessplanworkshops. Auch die Erstellung von Leitfäden und Handbüchern könnte in diesem Zusammenhang von Vorteil sein.

Grafik 10 Zufriedenheit mit verschiedenen Aspekten des Projektauswahlverfahrens, arithmetische Mittelwerte, nach Antragsphasen *)

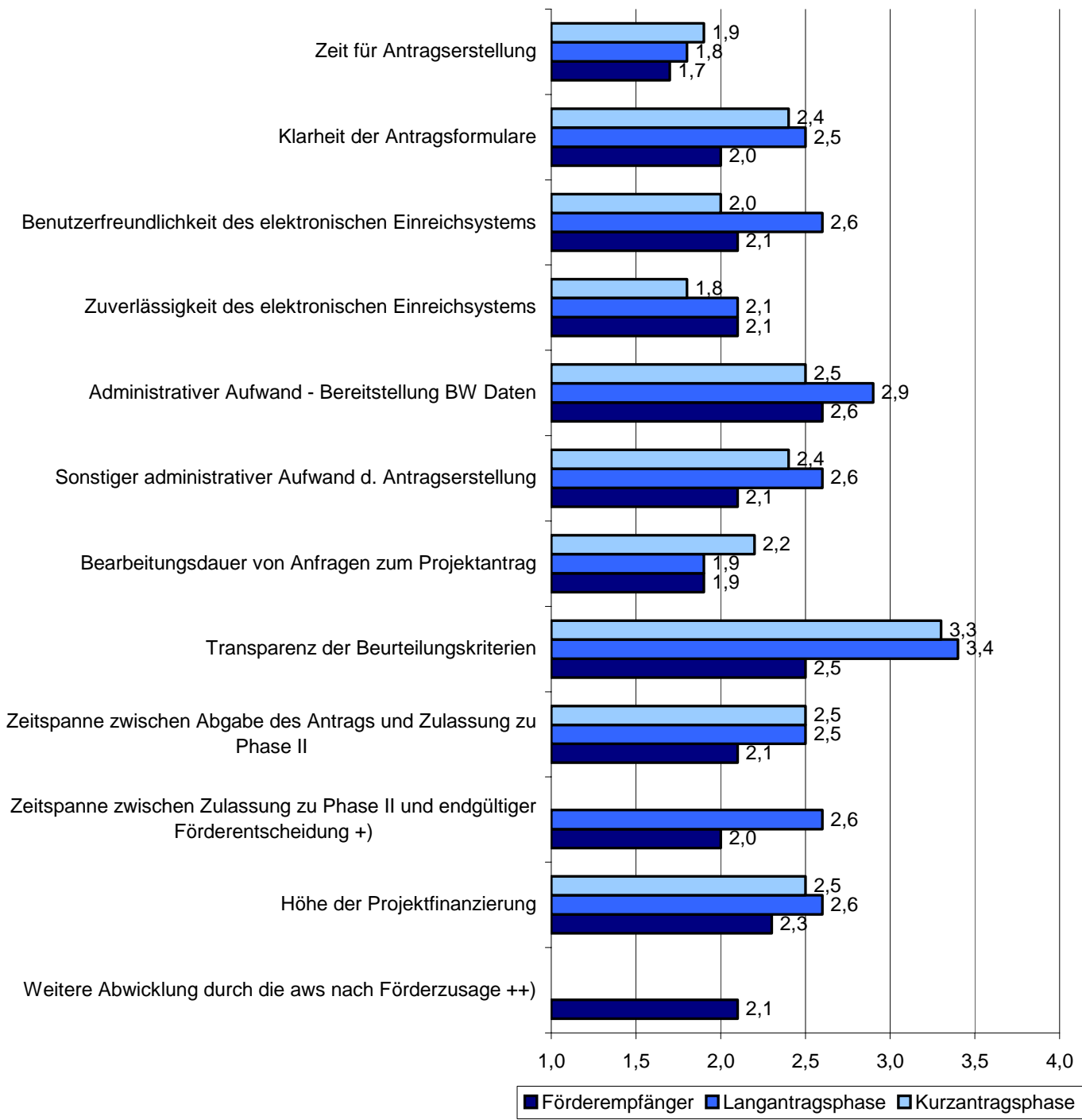

+) Diese Frage wurde den Unternehmen in der Kurzantragsphase nicht gestellt.

$++)$ Diese Frage wurde Unternehmen in der Kurz- und in der Langantragsphase nicht gestellt.

*) auf einer Skala von 1 = sehr zufrieden, 2 = zufrieden, 3 = weniger zufrieden, 4 = gar nicht zufrieden

Quelle: KMU FORSCHUNG AUSTRIA, N (FE) = 16, N (P2) $=17, \mathrm{~N}(\mathrm{P} 1)=48$. 
Deutlich negativ wurde der Aspekt der Transparenz im Projektauswahlverfahren bewertet (Durchschnittsnote: 3,2). Dies galt überraschenderweise nicht nur für die Unternehmen in der Langantragsphase, die keine positive Förderentscheidung erhalten haben (Durchschnittsbewertung: 3,4), sondern auch für die Förderempfänger, wo die Transparenz der am zweitschlechtesten bewertete Aspekt im Projektauswahlverfahren war (Durchschnittsnote: 2,5). Der Hauptgrund hierfür lässt sich vor allem in einer als ungenügend empfundenen Unternehmenskommunikation identifizieren. So wurden einerseits vor allem zu standardisierte Absagebriefe bemängelt, andererseits dürfte aber auch tlw. Unklarheit über die Programmzielsetzungen geherrscht haben. Entsprechende Unschärfen bei der Definition der Zielsetzungen (z. B. hinsichtlich des Stellenwertes des Kreativitätsaspektes in den Anträgen, siehe auch Abschnitt 3.1) kamen hier zum Tragen.

Es sollte allerdings auch berücksichtigt werden, dass eine „objektive“ Bewertung künstlerischer Elemente schwierig ist. Infolgedessen dürfte die Transparenz bei der Förderung der Kreativwirtschaft immer eine Herausforderung bleiben. Dies unterstreicht letztlich aber auch die Wichtigkeit transparenz-erhöhender Maßnahmen. Für iP dürften vor allem eine individualisiertere Unternehmenskommunikation, eine schärfere Definition und Vermittlung der Programmzielsetzungen sowie die bereits beschriebene Einbeziehung englischsprachiger Experten in den Jurys zur Erhöhung der Transparenz beitragen. Wenngleich Hinweise über zumindest wahrgenommene Doppelfunktionen oder Unvereinbarkeiten von Tätigkeiten (z. B. in den Jurys) nur vereinzelt, aber dennoch, zu vernehmen waren, sollten - als Grundlage für die glaubwürdige Vermittlung der Transparenz - in jedem Fall auch für die Zukunft potenzielle/tatsächliche Interessenskonflikte einzelner handelnder Personen vermieden werden.

\subsection{Begleitmaßnahmen im Rahmen von iP}

\subsubsection{Bisher angebotene Begleitmaßnahmen}

An Begleitmaßnahmen wurden im Rahmen des iP ImpulsProgramm creativwirtschaft bislang zwei ganztägige Businessplan-Workshops (am 31.3.2005 sowie am 5.4.2005) von der aws abgehalten. Die Workshops richteten sich an Betriebe, die sich für die Langantragsphase im 1. Call von iP qualifiziert haben und behandelten Themen wie den Aufbau und den Inhalt eines Businessplans, die Erstellung einer Plankostenrechnung sowie die Darstellung der Finanzierungssituation eines Betriebes. Darüber hinaus wurde auch ein Überblick über verschiedene Finanzierungsformen, wie sie z. B. auch die aws anbietet, gegeben und der Businessplan an Hand eines fiktiven Technologieunternehmens erläutert. Aus Zeitgründen wurde für die Vorträge auf bestehende Präsentationen im Bereich des Seedfinancingprogramms der aws zurückgegriffen. Die Teilnahme war für die Unternehmen mit keinerlei Kosten verbunden. An den Workshops haben insgesamt ca. 30 Personen aus 21 Projekten teilgenommen. ${ }^{6}$

Jene Betriebe, die nicht teilgenommen haben (nahezu ausschließlich Unternehmen, die nicht speziell für iP gegründet wurden und schon vor dem 1. Call bestanden haben), gaben hierfür vor allem an, dass sie bereits über genügend Vorkenntnisse verfügen (siehe Grafik 11). Jeweils etwa $38 \%$ konnten aus terminlichen Gründen (entweder auf Grund von Terminkollisionen oder weil sie allgemein zu wenig Zeit hatten) nicht teilnehmen. Sonstige Gründe (z. B. die räumliche Distanz) spielten für etwa $19 \%$ eine Rolle. Interessanterweise hat kein einziges der nichtteilnehmenden Unternehmen aus mangelndem Interesse von einem Besuch der Veranstaltungen abgesehen, was auf einen vorhandenen prinzipiellen Bedarf nach Aus- und Weiterbildungsmaßnahmen schließen lässt. Die Kommunikation der Workshoptermine dürfte gut funktioniert haben, da auch alle befragten NichtteilnehmerInnen über die Veranstaltungen informiert zu sein schienen.

\footnotetext{
${ }^{6}$ Vgl. ARGE iP, Statusbericht Oktober 2004 - April 2005, 2005.
} 
Grafik 11 Gründe für die Nicht-Teilnahme an den im Rahmen von iP angebotenen Businessplan-Workshops, Betriebe in Prozent

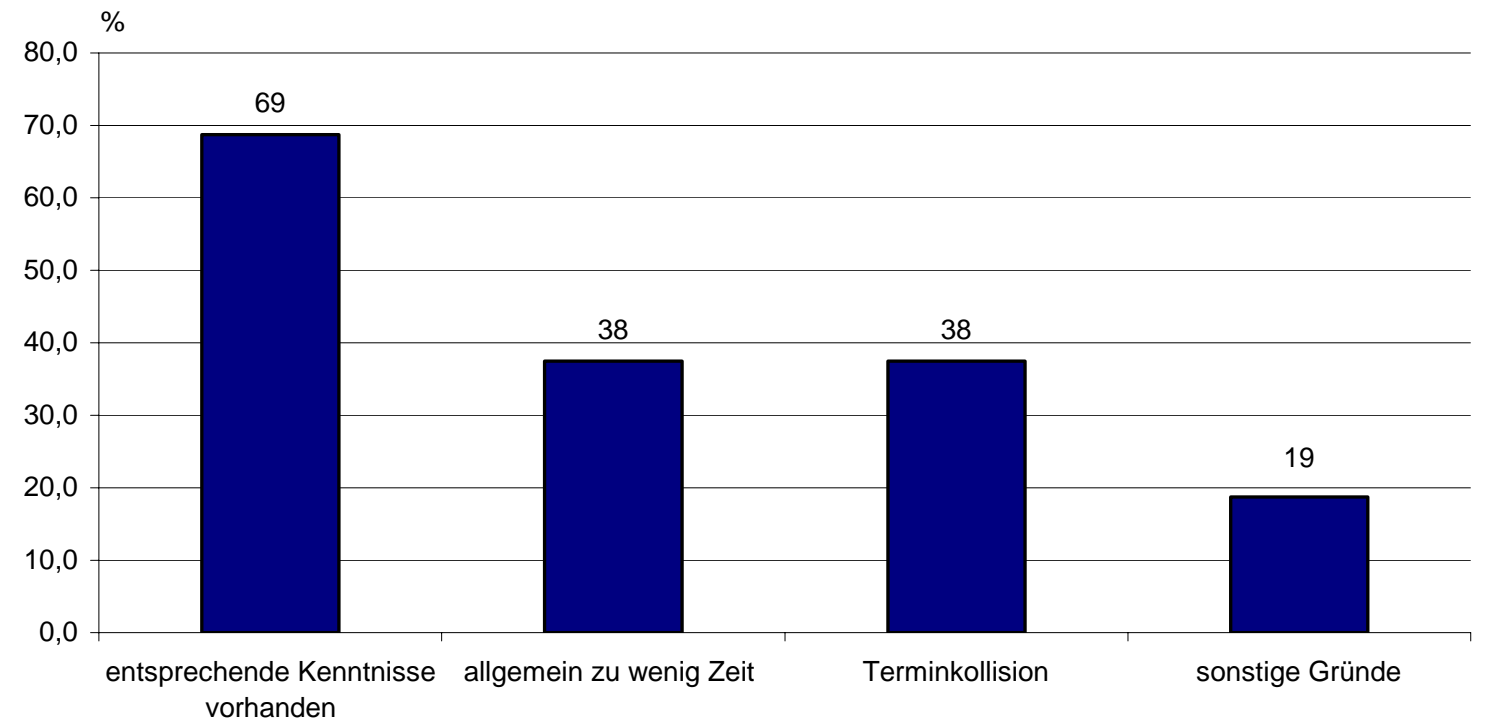

Quelle: KMU FORSCHUNG AUSTRIA, N = 16.

Die Gruppe der teilnehmenden Betriebe - die sowohl etablierte Unternehmen, deren VertreterInnen über betriebswirtschaftliche Vorkenntnisse verfügen, als auch Personen, für die die dargestellten Themenbereiche komplett neu waren, umfasste - hat die Workshops insgesamt positiv aufgenommen (siehe Grafik 12). Gut bewertet wurden vor allem der Zeitumfang der Veranstaltungen (Durchschnittsnote: 1,8 auf einer Skala von 1=sehr zufrieden bis 4=gar nicht zufrieden), die Qualität der Workshopunterlagen oder die erhaltenen Vorinformationen über den Workshop (Durchschnittsnote: jeweils 1,9). Nur unwesentlich schlechter wurden die Kompetenz der TrainerInnen (Note: 2,0), die Vermittlungsmethoden (Note: 2,1) und die Praxisrelevanz der Lehrinhalte (Note: 2,1 ) beurteilt. 


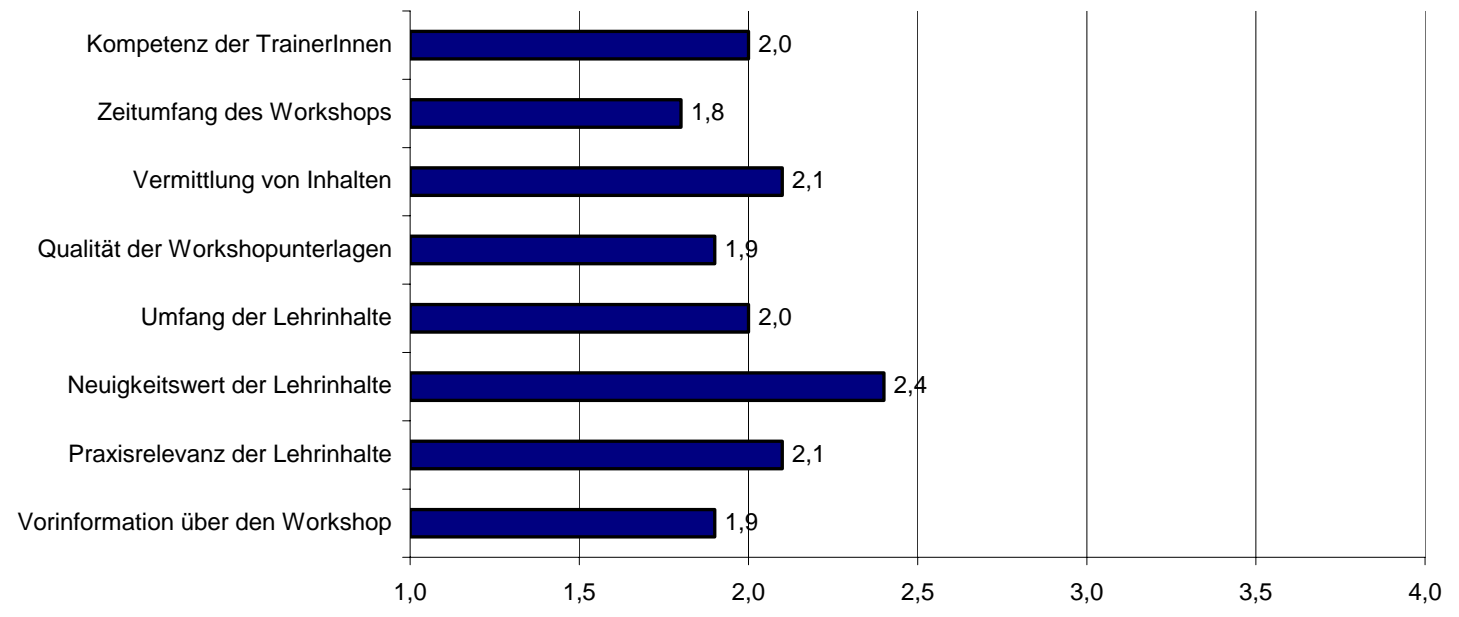

*) auf einer Skala von 1 = sehr zufrieden, 2 = zufrieden, 3 = weniger zufrieden, 4 = gar nicht zufrieden Quelle: KMU FORSCHUNG AUSTRIA, $N=10$

Etwas häufiger war hingegen der vermeintlich zu geringe Neuigkeitswert der Lehrinhalte ein Anlass zu Kritik (Durchschnittsnote: 2,4). In diesem Zusammenhang wurde vor allem vorgebracht, dass zu wenig Spezifika von Unternehmen in der Kreativwirtschaft Berücksichtigung fanden, z. B. bei der Wahl der Fallstudie oder auch bei der Wahl der verwendeten Tools (Windows Applikationen vs. den in der Kreativwirtschaft häufiger verwendeten Apple Macintosh Systemen).

\subsubsection{Bedarf nach Begleitmaßnahmen}

Wie sich bereits aus den Antworten der Workshopteilnehmerlnnen abgezeichnet hat (siehe weiter oben) besteht zum Teil erhebliches Interesse an Aus- und Weiterbildungsmöglichkeiten auf Seiten der am 1. Call von iP beteiligten Unternehmen (siehe Grafik 13) - vor allem wenn die Heterogenität der Einreicher (z. B: hinsichtlich bestehender und neu gegründeter Unternehmen) berücksichtigt wird. Nur etwa $20 \%$ der Betriebe äußerten keinerlei Wunsch hinsichtlich von iP beizubringender Aus- und Weiterbildungsmaßnahmen. Dies deckt sich auch mit den allgemeinen Erfahrungen der interviewten Experten in der Kreativwirtschaftsszene.

Etwa vier von zehn Unternehmen interessieren sich für entsprechende Angebote hinsichtlich rechtlicher Fragestellungen wie etwa Vertragsgestaltung oder die zunehmend aktueller werdenden Intellectual Property Rights (IPR) (siehe Grafik 13). Ein geringfügig kleinerer Anteil äußerte Weiterbildungsbedarf im Bereich der Gestaltung von Kooperationen. Nachfrage besteht des Weiteren auch für Weiterbildungsmaßnahmen im Themenfeld Marketing und Vertrieb.

Der Anteil an Betrieben, die sich für Maßnahmen im Bereich der Projektantragserstellung (31\%) und Businessplanerstellung (21\%) interessiert, erscheint - angesichts der von den Juroren festgestellten Schwachstellen der Anträge im 1.Call in genau diesen Punkten - gering. Dies dürfte (zusammen mit der geringen Nachfrage nach Angeboten für sonstige betriebswirtschaftliche Bereiche und für das Themengebiet Unternehmensführung) zumindest zu einem Teil dem Verständnis zu entspringen, dass sich, wie bereits erwähnt, viele in der Kreativwirtschaft tätigen Personen nicht als „klassische“ UnternehmerInnen sehen und möglicherweise daher auch gegenüber „klassisch“-wirtschaftlichen Themenbereichen ein geringeres Problembewusstsein entgegenbringen. 


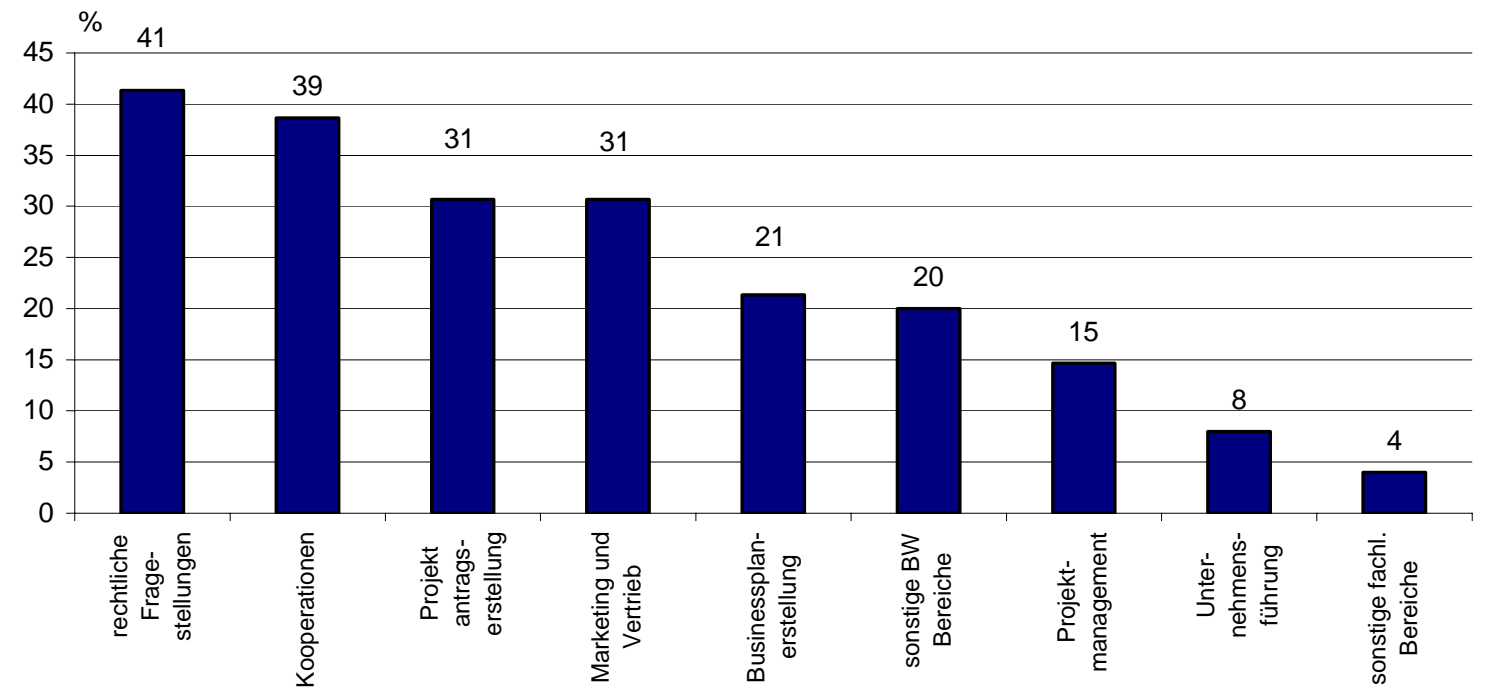

Quelle: KMU FORSCHUNG AUSTRIA, N = 75

Unter Berücksichtigung dieses Aspektes, eingeschränkter Budgetmittel und der Tatsache, dass für viele der angesprochenen Fachbereiche einschlägige Angebote für Weiterbildungsmaßnahmen zur Verfügung stehen ( $u$. a. vom WIFI, aber auch von anderen Einrichtungen) erscheint es für die Gestaltung des Begleitprogramms von iP sinnvoll, Themenschwerpunkte zu setzen. Die Gespräche mit Juroren und Experten sowie internationale Erfahrungen haben in dieser Hinsicht gezeigt, dass die Haupansatzpunkte Fragen der Projektantragsstellung/ Businessplanerstellung (hier speziell die Marktanalyse) sowie in weiterer Folge ein kontinuierliches Coaching in der Projektumsetzungsphase umfassen. Für die weiteren Themenbereiche gilt es zu prüfen, in wieweit bei Aus- und Weiterbildungsmaßnahmen anderer Institutionen und Programme Möglichkeiten bestehen, Brücken zu iP zu schlagen - dies speziell auch deswegen, da sich keine eindeutige Zahlungsbereitschaft der Unternehmen ( $29 \%$ wären nicht bereit, eine Kostenbeitrag zu übernehmen und $42 \%$ wissen nicht, ob sie bereit wären für Weiterbildungskurse und -workshops zu zahlen) aus der Befragung ableiten lässt. 


\section{Wirkungsanalyse}

\subsection{Kooperationsaktivitäten}

Eines der Ziele von iP, das auch in der Öffentlichkeitsarbeit deutlich unterstrichen wird, ist die Stärkung der betrieblichen Kooperationsaktivitäten in der Kreativwirtschaft, aber auch die Vernetzung der Unternehmen mit traditionellen Wirtschaftsbranchen. Im Folgenden soll nun der Frage nachgegangen werden, inwieweit iP bei den geförderten Projekten tatsächlich zu einer Erhöhung der Kooperationsaktivitäten geführt hat.

Ausgangspunkt der Analyse sind zunächst die bisherigen Kooperationsaktivitäten der einreichenden Betriebe. Diese sind in den letzten fünf Jahren, ihren eigenen Angaben zufolge, relativ häufig Kooperationen eingegangen (siehe Grafik 14). Dies trifft speziell auf andere Unternehmen als Kooperationspartner zu, mit denen rd. $79 \%$ der Betriebe häufig und $19 \%$ zumindest vereinzelt zusammengearbeitet haben. Ca. $47 \%$ kooperierten regelmäßig mit einzelnen KünstlerInnen, rd. $37 \%$ taten dies gelegentlich. Überraschend hoch ist auch die Kooperationsintensität mit wissenschaftlichen Einrichtungen (Anteil der häufig und vereinzelt kooperierenden Betriebe: rd. $30 \%$ bzw. rd. $55 \%$ ). Vergleichsweise weniger häufig wurde hingegen mit kulturellen und künstlerischen Institutionen, wie z. B. Museen, zusammengearbeitet.

Grafik 14 Häufigkeit, mit der bei iP einreichende Unternehmen in den vergangenen 5 Jahren Kooperationen eingegangen sind, nach Arten von Kooperationspartnern

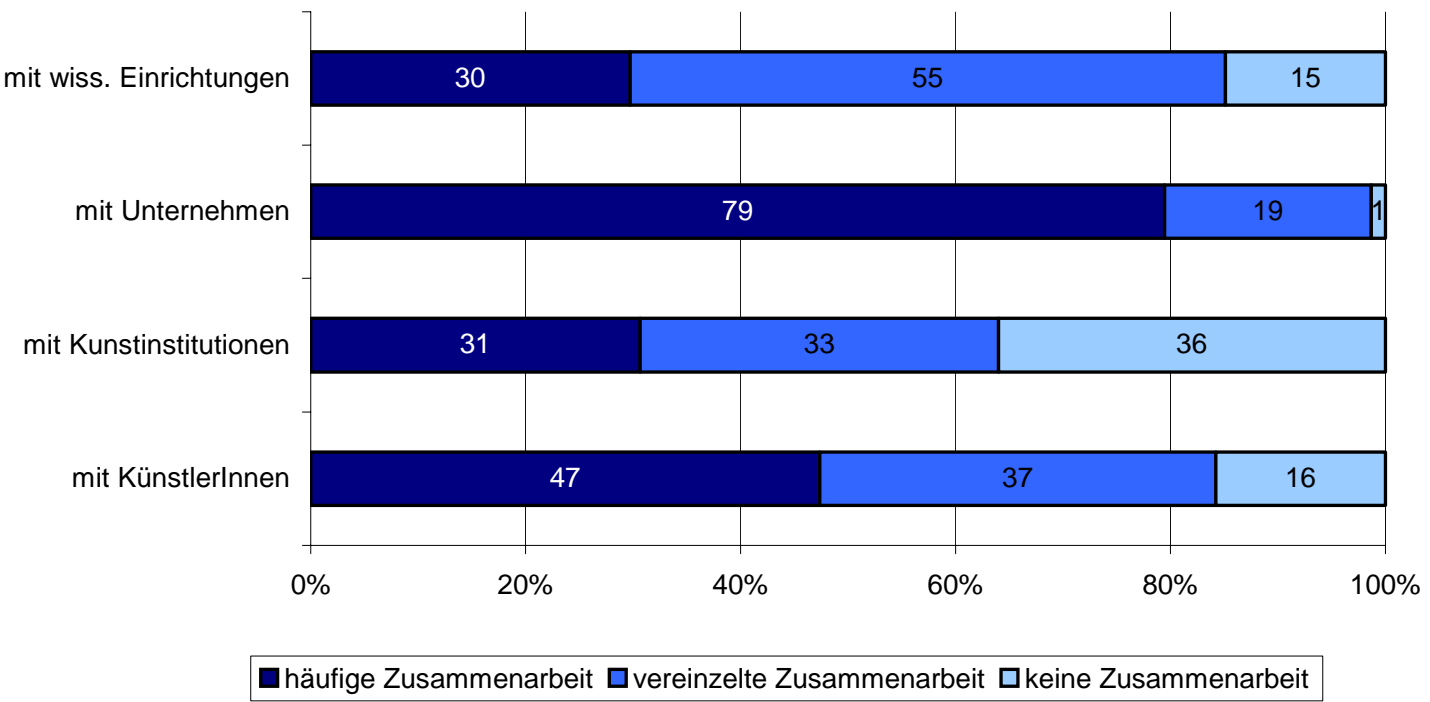

Quelle: KMU FORSCHUNG AUSTRIA, N = 75

Von den befragten Förderempfängern gaben alle an, im Rahmen ihres Projektes Kooperationen eingegangen zu sein, wobei die Qualität der Zusammenarbeit gut bis sehr gut sein soll. Etwa zwei Drittel der Förderempfänger haben bereits vor der Teilnahme an iP mit ihren derzeitigen Projektpartnern kooperiert. 
Angesprochen auf die Identität dieser Partner zeigt sich allerdings, dass die Unternehmen den Kooperationsbegriff sehr weit zu fassen scheinen und darunter tlw. auch klassische Kundenund Lieferantenbeziehungen verstehen. So gaben nur vier der 13 befragten Betriebe hinreichend konkrete Kooperationspartner an. Unter der nahe liegenden Annahme, dass die Betriebe in der Lang- und Kurzantragsphase ein ähnliches Kooperationsverständnis haben, wären die Werte aus Grafik 14 - im Hinblick auf Kooperationen, bei denen ein definiertes Konsortium von Betrieben im Rahmen eines Projektes an bestimmten Problemstellungen arbeitet - entsprechend zu relativieren.

Wird zudem die Tatsache berücksichtigt, dass das Kooperationsziel auf Maßnahmenebene nur als eines von sieben Gewichtungskriterien seine Entsprechung findet, ist ein Effekt, dass iP „netto“ (im Sinne einer Additionalität) Kooperationen gefördert hat, schwer abzuleiten. In der Folge stellt sich die Frage, ob die Intensivierung der Kooperationsaktivitäten im Sinne einer möglichen Zielüberfrachtung ein primäres Programmziel bleiben sollte. Alternativ könnte überlegt werden, ob eine Erhöhung der Sensibilisierung zum Thema Kooperationen als sekundäres Ziel der instrumentellen Ausgestaltung von iP (unter Beibehaltung der Kooperationen als Gewichtungskriterium bei Projektanträgen) und dem generellen Kooperationsverständnis in der Kreativwirtschaft nicht eher gerecht werden würde.

\subsection{Outputs und Additionalität}

Auf Grund der geringen Zeitspanne zwischen der Förderzusage und der durchgeführten Unternehmensbefragung bestehen noch keine Projektoutputs, die als solche erhoben werden könnten. Als Referenz für eine Ex-Post Analyse ist es jedoch durchaus von Interesse, die diesbezüglichen Erwartungen der Unternehmen im Rahmen der vorliegenden begleitenden Evaluierung zu untersuchen. Entsprechende Outputindikatoren wurden der Stiftung seitens iP für Evaluierungen auch vorgeschlagen.

Tabelle $4 \quad$ Geplante marktorientierte Outputs in den iP Projekten

\begin{tabular}{l|c}
\hline \multicolumn{1}{c|}{ Output } & Anzahl \\
\hline Neue Produkte & $>13$ \\
Neue Verfahren & $>4$ \\
Prototypen („Proof of Concept“) & $>9$ \\
Neue Dienstleistungen & $>12$ \\
Neue Vermarktungsinstrumente & $>9$ \\
\hline Quelle: KMU FORSCHUNG AUSTRIA & \\
\hline
\end{tabular}

Geht man von den Angaben der insgesamt 16 antwortenden Unternehmen als Untergrenze für den Output des 1. Calls aus, so sollen im Rahmen der Projekte mindestens 13 neue Produkte, vier neue Verfahren, neun Prototypen oder „Proof of Concept“-Studien, 12 neue Dienstleistungen und neun neue Vermarktungsinstrumente entwickelt werden (siehe Tabelle 4). Diese Zielgrößen sind in ihrer Gesamtheit, unter Berücksichtigung der Zahl der Projekte und der Projektvolumina, durchaus als ambitioniert zu bezeichnen. 


\begin{tabular}{l|c}
\hline \multicolumn{1}{c|}{ Output } & Anzahl \\
\hline Anmeldung neuer Patente & $>12$ \\
Anmeldung neuer Marken & $>8$ \\
Begründung von Urheberrechten & $>3$ \\
Neue Arbeitsplätze & $>22$ \\
\hline
\end{tabular}

Quelle: KMU FORSCHUNG AUSTRIA

Im Bereich der Intellectual Property Rights werden zumindest 12 Patentanmeldungen angestrebt, was sicherlich - im Vergleich zum Patentoutput anderer Förderprogramme - eine sogar noch größere Herausforderung darstellen wird (siehe Tabelle 5). Realistischerweise wird die Anzahl neuer Patente wohl deutlich geringer ausfallen. Weiters ist geplant, ein Minimum an acht neuen Marken anzumelden und drei oder mehr Urheberrechte zu begründen. Die Beschäftigungseffekte dürften sich - absolut betrachtet - mit 22 zusätzlichen Arbeitskräften in Grenzen halten.

Es bestehen Hinweise darauf, dass der 1. Call von iP hohe Additionalitätseffekte aufweist (siehe Grafik 15). Kein einziger der 13 auf diese Frage antwortenden Förderempfänger hätte das Projekt ohne Förderung in vollem Umfang und ohne Zeitverzögerung durchgeführt. Drei Unternehmen hätten ihr Vorhaben zumindest großteils auch ohne Unterstützung durch iP in Angriff genommen, während der Umfang der Projekte bei weiteren zwei signifikant geringer ausgefallen wäre. Bei etwa vier Betrieben wäre es ohne Förderung zu Zeitverzögerungen gekommen, die bei der Hälfte dieser Betriebe auch mit deutlich reduzierten Projektumfängen einhergegangen wären. Reine Additionalitätseffekte (keinerlei Durchführung des Projektes ohne Förderung) können bei vier Betrieben ausgemacht werden.

Einschränkend muss hinzugefügt werden, dass zum Einen keine adäquate Kontrollgruppe für Vergleichszwecke zur Verfügung gestanden ist und zum Anderen, dass die Stichprobengröße im Verhältnis zur Gesamtzahl der Projekte zu klein ist, um eine gesicherte Aussage für den gesamten Call tätigen zu können. Dementsprechend können die zuvor angeführten Ergebnisse nur indikativ gesehen werden. 


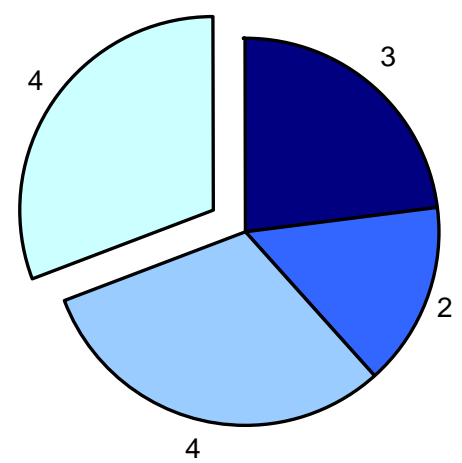

Durchführung des Projektes im überwiegenden Ausmass trotz Förderung 口Durchführung des Projektes trotz Förderung, aber im geringeren Ausmass 口Durchführung des Projektes trotz Förderung, aber mit zeitlicher Verzögerung 口keine Durchführung des Projektes ohne Förderung

*) Antworten auf die Frage: Hätten Sie das Projekt auch ohne Förderung durch iP durchgeführt? Quelle: KMU FORSCHUNG AUSTRIA, N = 13

Neben dem finanziellen Aspekt sehen viele Betriebe einen Zusatznutzen von iP in einer gesteigerten Publicity bzw. einem besseren Image des Unternehmens. Hier drückt sich eine hohe Erwartungshaltung bei der Zielgruppe im Hinblick auf zukünftige Tätigkeiten und der Reputation von iP aus, was als Chance bzw. Herausforderung für das iP Team gesehen werden sollte.

Etwa 64 \% der EinreicherInnen würden im Rahmen eines weiteren Calls von iP wieder um eine Förderung ansuchen. Erwartungsgemäß ist der Anteil der „Wiedereinreichwilligen“ unter den Förderempfängern am größten (93 \%), fällt aber nichtsdestotrotz auch bei den Unternehmen in der Kurzantragsphase (ca. $54 \%$ ) und in der Langantragsphase (rd. $65 \%$ ) relativ hoch aus. Zusammen mit den etwa $64 \%$ der antwortenden Interessenten, die sich eine Einreichung zu einem späteren Zeitpunkt vorstellen könnten, dürfte dies auf eine rege Beteiligung bei späteren Calls hindeuten. 


\section{Schlussfolgerungen und Lerneffekte}

Die Evaluierungsergebnisse legen den Schluss nahe, dass das iP ImpulsProgramm creativwirtschaft die zugedachte Rolle als Impulsbringer für die österreichische Creativ Industries Community weitgehend erfüllt und in einigen Bereichen (so z. B: bei der Zahl der Einreichungen) die Erwartungen auch übertrifft. Positiv bewertet werden vor allem das generelle Programmmanagement, wie z. B. die Öffentlichkeitsarbeit, aber auch die meisten Aspekte des Projektauswahlverfahrens (hier nicht zuletzt die Wahl und Ausgestaltung des Calls als kompetitives Auswahlinstrument). Inhaltlich dürften vor allem die Projekte aus dem Bereich Multimedia überzeugen. Ein Erfolgsfaktor dürfte auch sein, dass sowohl bestehende als auch neu gegründete Unternehmen gefördert werden. Schließlich bestehen auch Hinweise darauf, dass das Programm über vergleichsweise hohe Additionalitätseffekte verfügt.

Optimierungsmöglichkeiten zeigen sich einerseits in Detailbereichen (wobei viele der Detailprobleme im Rahmen der Etablierung der Expertenbeiräte (die für sich betrachtet begrüßenswert ist) bereits aufgegriffen und im anlaufenden zweiten Call beseitigt wurden, z. B. hinsichtlich der Schwächen des elektronischen Einreichsystems. Andererseits scheint aber auch mit der notwendigen Erhöhung der Transparenz im Projektauswahlverfahren eine zentrale Herausforderung für das Programm zu bestehen.

Im Folgenden sollen nun Maßnahmenvorschläge zu den identifizierten Problemfeldern sowie Erweiterungs- und Optimierungspotenziale diskutiert werden:

\section{Prüfung von Möglichkeiten zur Erhöhung der Transparenz}

Die angesprochene Problematik der Transparenz im iP ImpulsProgramm creativwirtschaft scheint sich aus drei Faktoren heraus zu ergeben: Erstens, dass Ziele und Zielgruppen tlw. im Vorfeld der Antragserstellung nicht hinreichend klar vermittelt wurden. Dies betrifft speziell den Stellenwert der kreativ-gestalterischen Elemente im Verhältnis zum Innovationsgrad, aber auch die Zielsystematik, wo vereinzelt Zielen nicht ausreichend eindeutig Maßnahmen und Instrumente gegenüberstehen. Zweitens zeigte sich, dass die dargelegten Ablehnungsgründe vielfach den Informationsbedarf der Betriebe nicht abgedeckt haben. Der dritte Faktor erklärt sich allgemein mit den Schwierigkeiten, künstlerisch-kreative Elemente „objektiv“ zu bewerten. In dieser Hinsicht ergeben sich noch höhere Ansprüche an die Transparenz im Projektauswahlverfahren als bei anderen Innovationsförderprogrammen.

Bislang zeigt die vergleichsweise negative Bewertung des Transparenzaspektes durch die Unternehmen nur geringe Auswirkungen auf die Zufriedenheit mit dem Gesamtprogramm, die Förderung durch iP wird meist als Qualitätssiegel wahrgenommen. Speziell vor dem Hintergrund des zuvor beschriebenen thematischen Faktors sollte aber trotzdem jede Möglichkeit zur Erhöhung der Transparenz des Programms geprüft werden. Andernfalls würde die Gefahr bestehen, dass sich die Schwäche mittelfristig im Ruf des Programms manifestiert - mit entsprechenden Auswirkungen auf die Qualität und die Anzahl von Einreichungen.

Ein wichtiger Faktor zur glaubwürdigen Vermittlung der Transparenz in Förderprogrammen ist die Expertise und Unbahängigkeit der Jurymitglieder und der entscheidungsbefugten Personen. Wenngleich diesbezügliche Hinweise über zumindest wahrgenommene Doppelfunktionen oder Unvereinbarkeiten von Tätigkeiten nur vereinzelt, aber dennoch, zu vernehmen waren, sollten als Grundlage für die glaubwürdige Vermittlung der Transparenz - in jedem Fall auch für die Zukunft potenzielle/tatsächliche Interessenskonflikte einzelner handelnder Personen vermieden werden. 


\section{Stärkere Einbeziehung internationaler Expertise}

Hinsichtlich der Juryzusammensetzungen wurde, um Unabhängigkeit und Kompetenz zu garantieren, auf eine Mischung internationaler und nationaler ExpertInnnen aus dem deutschsprachigen Raum zurückgegriffen, was prinzipiell positiv zu bewerten ist. Das Standing der Jury ließe sich aber weiter ausbauen, würde man auch englischsprachige Experten (speziell aus Großbritannien, das im Bereich der Kreativwirtschaft führend ist) einbeziehen. Dazu müssten Anträge jedoch zwangsläufig in Englisch verfasst werden. Auch die Kosten zur Abgeltung der JurorInnentätigkeit würden entsprechend höher ausfallen. Der Nutzen könnte aber den zusätzlichen Aufwand deutlich übertreffen, nicht zuletzt auch wegen der gesteigerten Sichtbarkeit der Projekte im internationalen Kontext (womit man gleichzeitig auch dem Kritikpunkt der zu geringen Marktorientierung in den Anträgen entgegenwirken würde). Das Verfassen der Anträge in englischer Sprache sollte zumindest den Betrieben im Multimediabereich zugetraut werden können, die bei der Businessplan- und Antragserstellung wohl die meiste Erfahrung mitbringen. Alternativ könnte überlegt werden, englischsprachige Experten verstärkt in den Beiräten einzubeziehen.

\section{Verbesserungspotenzial in der Unternehmenskommunikation}

Ein weiterer wesentlicher Ansatzpunkt bildet die Kommunikation mit den Unternehmen. Es bestehen Hinweise darauf, dass Zielsetzungen, Zielgruppen und konkrete Programminhalte im Rahmen der PR Tätigkeiten schärfer kommuniziert werden könnten. Die durchaus zulässige Betonung des kreativen Charakters des Förderprogramms (durch eine künstlerische Gestaltung der PR Unterlagen) sollte nicht zulasten des Informationsgehaltes gehen. Für die Illustration dessen, wen und was iP fördern will, würden sich „Good Practice“ Beispiele anbieten. Bei abgelehnten Anträgen könnte überlegt werden, inwieweit Möglichkeiten zu einer höheren Personalisierung/Individualisierung der Absagebriefe bestehen bzw. ob weitere, formalisierte Feedbackschleifen zu den Unternehmen in sinnvoller Weise implementiert werden können.

\section{Erhöhte Kooperationsintensität nur als sekundäres Ziel}

Grundvoraussetzung für eine erfolgreiche Programmdurchführung ist, dass Ziele und Maßnahmen ausreichend klar definiert und zueinander in Beziehung gesetzt sind. Im (insgesamt stimmigen und gut strukturierten) Ziel- und Maßnahmensystem von iP lassen sich in dieser Hinsicht zwei Detailproblembereiche identifizieren: Das eine Problemfeld bildet die nicht ausreichend erscheinende Umsetzung des Kooperationsziels auf Maßnahmenebene. Bei den bislang im Rahmen von iP geförderten Projekten scheint es sich um keine Kooperationsprojekte zu handeln, wie sie z. B. Gegenstand eines hierfür dezidierten Instrumentariums (Stichwort: Verbundprojekte oder Cluster) sind. Grund hierfür ist in erster Linie, dass die Kooperationsbildung keine Voraussetzung für die Zulassung des Antrags, sondern nur ein Gewichtungskriterium im Projektauswahlverfahren war. Eine Erhöhung der Kooperationsintensität, die auf die Förderung durch iP zurückzuführen wäre, ließ sich bei den Fördernehmern nicht feststellen. In der Folge stellt sich daher die Frage, ob es nicht sinnvoll wäre, Kooperationen nur als sekundäres Ziel, z. B. in Richtung Sensibilisierung der Unternehmen für die Kooperationsproblematik, zu definieren. Dies dürfte der derzeitigen instrumentarischen Ausgestaltung von iP eher gerecht werden.

\section{Mehr Mut zur Betonung des Kreativitätsaspektes}

Das zweite Problemfeld betrifft die oben bereits angeführte Bewertung des kreativ-künstlerischen Gehaltes der Projekte. Zwar grenzt sich iP deutlich von einer reinen Kunstförderung ab und orientiert sich eher an technologischen Inhalten, dennoch ist es gerade der Kreativitätsaspekt, der iP von anderen Innovationsförderprogrammen abhebt und der auch seitens des Programmmanagements betont wird. Eine, bisweilen im 1. Call kritisierte, zu starke Orientierung an technologischen Inhalten könnte zur Folge haben, dass die Zielgruppe der Kreativen, sich in den geförderten Projekten nicht wieder findet und bei zukünftigen Calls von Einreichungen absieht. In diesem Sinne erscheint es zweckmäßig, dass geförderte Projekte einen eindeutigen Bezug zur Kreativwirtschaft haben bzw. eindeutige kreative Elemente beinhalten. 


\section{Zielgruppenspezifisches Vorgehen in den einzelnen Themenbereichen}

Die qualitätsmäßigen Schwankungen der Anträge zwischen den drei Themenbereichen Musik, Design und Multimedia sind, Experten zufolge, auf unterschiedliche strukturelle Gegebenheiten in diesen Bereichen und auf einen unterschiedlichen unternehmerischen Background zurückzuführen. Im Sinne dessen, dass iP in allen drei Bereichen eine Impulsfunktion ausüben soll (und kein Bias in Richtung des am weitest entwickelten Sektors auftritt) wäre zu überprüfen, inwieweit ein zielgruppen-/themenspezifisches Vorgehen, z. B. hinsichtlich der Strenge der anzuwendenden Beurteilungskriterien, angebracht wäre. Dies betrifft vor allem die wirtschaftlichen Kriterien, deren Erfüllung für viele Unternehmen mit zu viel Aufwand verbunden war. Alternativ könnte auch überlegt werden, „Sondercalls“ auszuschreiben, wo die wirtschaftlich-formalen Hürden noch niedriger angesetzt werden und die künstlerische Idee noch deutlicher im Vordergrund steht. Letzteres ist aber mit auch mit deutlichen Risiken verbunden, speziell hinsichtlich der Definition der nichtsdestotrotz notwendigen Mindeststandards. Die Fokussierung auf die drei Themenbereiche sollte in regelmäßigen Abständen überprüft werden. Experten verweisen darauf, dass - vor allem im internationalen Kontext - Schnittstellenprojekte zwischen den drei Bereichen an Bedeutung gewinnen.

\section{Begleitmaßnahmen}

Im Bereich der Begleitmaßnahmen dürfte erhebliches Ausbaupotenzial bestehen, das sich aus einer breit artikulierten Nachfrage nach Aus- und Weiterbildungsmaßnahmen durch die Unternehmen und aus den identifizierten Schwächen der Anträge im 1. Call ergibt. Die thematische Breite an gewünschten Begleitmaßnahmen lässt eine Priorisierung unerlässlich erscheinen. Zu prüfen wäre jedenfalls, inwieweit Synergiepotenziale mit den Kursangeboten bestehender Ausund Weiterbildungsinstitutionen bestehen bzw. inwieweit deren Angebot die Spezifika der Kreativwirtschaft berücksichtigen. Angesichts der Schwächefelder in den Anträgen dürften sich für iP vor allem Begleitmaßnahmen in den Bereichen Antragsstellung/Businessplanerstellung sowie Projektcoaching (in der Umsetzungsphase) anbieten. 


\section{Liste der InterviewpartnerInnen}

\begin{tabular}{|c|c|c|c|}
\hline & Name & Institution & Funktion \\
\hline \multicolumn{4}{|c|}{ Programmverantwortliche $^{7}$} \\
\hline 1 & Sonja Hammerschmid & aws & $\begin{array}{l}\text { Geschäftsführerin iP ImpulsProgramm } \\
\text { creativwirtschaft }\end{array}$ \\
\hline 2 & Christian Atzmüller & $\begin{array}{l}\text { WKO / arge creativ } \\
\text { wirtschaft }\end{array}$ & $\begin{array}{l}\text { Geschäftsführer iP ImpulsProgramm } \\
\text { creativwirtschaft }\end{array}$ \\
\hline 3 & Sabine Pümpel & iP & Projektleitung iP \\
\hline 4 & Karl Schiller & aws & Businessplanworkshop \\
\hline 5 & Carina Felzmann & Cox Orange & Mitglied des ARGE Rates \\
\hline \multicolumn{4}{|c|}{ iP Jury } \\
\hline 6 & Susanne Kirchmayr & & DJ \\
\hline 7 & Norbert Kettner & $\begin{array}{l}\text { departure wirtschaft, } \\
\text { kunst und kultur } \\
\text { GmbH }\end{array}$ & Geschäftsführer \\
\hline 8 & Kurt Leutgeb & Aws & $\begin{array}{l}\text { Bereichsleiter/Prokurist Förderung \& } \\
\text { Finanzierung }\end{array}$ \\
\hline 9 & Oliver Michel & $\begin{array}{l}\text { c/o Geschäftsart } \\
\text { GmbH, Office Berlin }\end{array}$ & Geschäftsführer \\
\hline 10 & Andi Pongratz & $\begin{array}{l}\text { X-Art ProDivision } \\
\text { GmbH }\end{array}$ & Geschäftsführer \\
\hline 11 & Peter Rantasa & $\begin{array}{l}\text { Mica - music } \\
\text { information center } \\
\text { austria }\end{array}$ & Geschäftsführer \\
\hline 12 & Axel Thallemer & $\begin{array}{l}\text { Kunstuniversität Linz } \\
\text { Industrial Design }\end{array}$ & Professor, Institutsleiter \\
\hline 13 & Herbert Winkler & Creative Solutions & Geschäftsführer \\
\hline \multicolumn{4}{|c|}{ ExpertInnen } \\
\hline 14 & Friedrich Gnad & StadtArt & Geschäftsführer \\
\hline 15 & Erich Pötschacher & Instinct Domain & Geschäftsführer \\
\hline 16 & Michael Stampfer & WWTF & Leiter \\
\hline 17 & Sian Prime & Nesta (UK) & $\begin{array}{l}\text { Training \& Development Manager of the } \\
\text { Creative Pioneer Programme }\end{array}$ \\
\hline
\end{tabular}

\footnotetext{
${ }^{7}$ Bei den Programmverantwortlichen bezieht sich die Spalte Funktion auf die Tätigkeiten im Rahmen von iP.
} 